\title{
Ultra-Low Cycle Fatigue Tests and Fracture Prediction Models for Duplex Stainless Steel Devices of High Seismic Performance Braced Frames
}

\author{
Marco Baiguera ${ }^{1}$, George Vasdravellis ${ }^{2}$, and Theodore L. Karavasilis ${ }^{3}$ \\ ${ }^{1}$ Research Associate. Department of Civil, Environmental and Geomatic Engineering, University College \\ London, London WC1E 6BT, UK. \\ ${ }^{2}$ Associate Professor. Institute for Infrastructure and Environment, Heriot-Watt University, Edinburgh EH14 \\ 4AS, UK (corresponding author). Email: g.vasdravellis@ hw.ac.uk \\ ${ }^{3}$ Professor of Structures and Structural Mechanics. Faculty of Engineering and the Environment, University of \\ Southampton, Southampton SO17 1BJ, UK.
}

\section{ABSTRACT}

This paper presents ultra-low cycle fatigue tests and the calibration of different fracture models for duplex stainless steel devices of high seismic performance braced frames. Two different geometries of the devices were tested in full-scale under fourteen cyclic loading protocols up to fracture. The imposed protocols comprised of standard, constant amplitude, and randomly-generated loading histories. The test results show that the devices have stable hysteresis, high post-yield stiffness, and large energy dissipation and fracture capacities. Following the tests, two micromechanics-based models, i.e. the Cyclic Void Growth Model and the built-in Abaqus ductile fracture model, were calibrated using monotonic and cyclic tests on circumferentially-notched coupons and complementary finite element simulations. In addition, Coffin-Manson-like relationships were fitted to the results of the constant amplitude tests of the devices and the Palmgren-Miner's rule was used to predict fracture of the devices under the randomly generated loading protocols. Comparisons of the experimental and numerical results show that the calibrated models can predict ductile fracture of the devices due to ultra-low cycle fatigue with acceptable accuracy.

\section{INTRODUCTION}

A modern seismic design philosophy is to isolate damage in steel energy dissipation devices and protect the main structural members from yielding with the aid of capacity design rules. 
Energy dissipation devices can be designed to be easily accessible and replaceable (if needed) so that repair costs and downtime in the aftermath of strong seismic events can be significantly reduced (Soong and Spencer 2002; Symans et al. 2008). Steel yielding devices have stable and predictable hysteretic behavior and are insensitive to ambient temperature variations. Based on the first concepts developed in New Zealand in the 1970s (Kelly et al. 1972; Skinner et al. 1975), a wide range of steel yielding devices have been proposed for beam-column connections, braces, and base isolation systems. Early developments include the U-strip hysteretic dampers and devices made of multiple plates with optimized shape. Examples of the latter are the added damping and stiffness (ADAS) damper (Steimer et al. 1981; Whittaker et al. 1991) and the triangular-plate added damping and stiffness (T-ADAS) damper (Tsai et al. 1993). Other examples include the honeycomb damper used as seismic isolation system in bridges (Kajima 1991), C-shaped and E-shaped hysteretic dampers for bridges (Ciampi and Marioni 1991; Marioni 1997; Tsopelas and Constantinou 1997), slit-type dampers applied to beam-column connections or brace members (Chan and Albermani 2008; Oh et al. 2009), yielding shear panels (Nakashima et al. 1994), and cast-iron yielding fuses installed in braces (Gray et al. 2010). Steel cylindrical pins with hourglass-shape bending parts were used as the energy dissipation mechanism of a steel post-tensioned beam-column connection for self-centering moment-resisting frames (Vasdravellis et al. 2013a; Vasdravellis et al. 2013b).

A critical failure mode of steel yielding devices is ductile fracture. Under seismic loading, fracture of metals typically occurs after a relatively small number of cycles accompanied by large-scale plasticity. This loading is often termed as ultra-low cycle fatigue (ULCF). Nip et al. (2010) conducted low-cycle fatigue and ULCF tests on different structural steel grades, i.e. carbon steel and austenitic stainless steel, and found that the ductile fracture occurs when the number of cycles is below 100. Several studies have demonstrated that the fracture 
mechanism for ULCF is similar to monotonic ductile fracture, since it involves cyclic growth and collapse of voids (Nip et al. 2010; Kanvinde 2017). Therefore, micromechanics-based approaches that originate from fracture models for monotonic loading have been recently proposed to predict ULCF fracture (Kanvinde and Deierlein 2007; Myers et al. 2010; Jia and Kuwamura 2015; Wen and Mahmoud 2016b; Smith et al. 2017).

Vasdravellis et al. (2014) investigated the ductile fracture behavior of hourglass-shaped pins made of different steel grades, i.e. high-strength steel, austenitic stainless steel, and duplex stainless steel. The results showed that duplex stainless steel pins, named as SSPs, have the most desirable behavior for seismic design purposes, as they exhibit excellent ductility, high post-yield stiffness, and large fracture capacity. The notably high post-yield stiffness of the SSPs was utilized to reduce the residual drifts in a dual concentrically-braced momentresisting frame (CBF-MRF) proposed by Baiguera et al. (2016), where SSPs are installed in series with the braces. Nonlinear dynamic analyses of the dual CBF-MRF showed that the high post-yield stiffness of the SSPs results in negligible residual drifts under the Design Basis Earthquake (DBE, $10 \%$ probability of exceedance in 50 years) and very small residual drifts under the Maximum Considered Earthquake (MCE, 2\% probability of exceedance in 50 years). In the assessment of the CBF-MRF, ductile fracture of SSPs was preliminarily evaluated based on the tests conducted in Vasdravellis et al. (2014). However, the available experimental data referred to a limited number of cyclic tests conducted under one-sided loading protocols.

This paper presents the results of an experimental investigation on the seismic performance of full-scale SSPs under full-cycle ULCF loading protocols. This study aims to provide calibrated models for predicting ductile fracture of the SSPs, which can be implemented in seismic collapse evaluation of buildings equipped with such devices. The results of fourteen cyclic tests on two full-scale SSP geometries, selected from the prototype dual CBF-MRF 
proposed in Baiguera et al. (2016), are presented. The tests were conducted using a testing apparatus reproducing the SSP-brace connection, and various loading histories, i.e. standard, constant amplitude (CA), and randomly-generated protocols. Following the tests, two micromechanics-based fracture models, i.e. the CVGM and the built-in Abaqus ductile fracture model, were calibrated for SSD using tests on circumferentially-notched specimens (CNSs) and complementary simulations using the finite element method (FEM). CoffinManson-like relationships were fitted to the CA tests and were used to predict fracture of the SSPs under the random loading protocol tests, in combination with a Palmer-Miner linear damage accumulation rule. The ability of the models to predict fracture was assessed against the experimental tests of SSPs.

\section{PROTOTYPE FRAME}

Fig. 1(a) shows the CBF-MRF proposed by Baiguera et al. (2016). The SSPs are installed in series with the braces and pass through aligned holes between the gusset plate and a strong Ushaped plate, which is connected by either welding or bolting to the brace member [Fig. 1(b)]. The SSPs dissipate energy due to inelastic bending perpendicular to their axis. The geometric properties of a SSP are shown in Fig. 2(a). The bending hourglass parts have length $L_{\mathrm{SSP}}$, external diameter $D_{\mathrm{e}}$, and mid-length diameter $D_{\mathrm{i}}$. The hourglass shape promotes a constant curvature and a uniform distribution of plastic deformations along the length of the SSP, delaying in that way fracture. The design of a SSP includes the selection of $D_{\mathrm{e}}, D_{\mathrm{i}}$ and $L_{\mathrm{SSP}}$ to provide the required force $F_{\mathrm{SSP}}$ and to ensure a ductile flexural rather than a nonductile shear failure. A detailed design procedure for SSPs is given in Vasdravellis et al. (2014) and is not repeated herein. To meet capacity design requirements and avoid undesirable column failure due to high post-yield stiffness of the SSPs, friction pads are placed between the brace members and the beam gusset plate at the top of each floor [Fig. 1(a)]. The friction pads are activated at a predefined story drift level. More details on the 
geometry and seismic performance of the proposed dual CBF-MRF are presented in Baiguera et al. (2016).

\section{EXPERIMENTAL PROGRAM}

108

109

\section{Specimens}

In the dual CBF-MRF, each SSP-brace connection is made of four or more identical SSPs that work in parallel to resist the brace axial force [Fig. 1(b)]. Since all SSPs undergo the same displacement when loaded, tests were conducted on a single SSP. Two different SSP geometries were tested in full-scale, representing the devices at the third and sixth story of the prototype building, and denoted as SSP1 and SSP2, respectively. SSP1 has $D_{\mathrm{e}}=50 \mathrm{~mm}, D_{\mathrm{i}}=$ $24 \mathrm{~mm}$ and $L_{\mathrm{SSP}}=225 \mathrm{~mm}$, while SSP2 has $D_{\mathrm{e}}=40 \mathrm{~mm}, D_{\mathrm{i}}=18 \mathrm{~mm}$ and $L_{\mathrm{SSP}}=225 \mathrm{~mm}$. The two geometries are shown in Fig. 2(b).

Seven specimens of each geometry were manufactured by machining $740 \mathrm{~mm}$ long round rolled bars, having diameters equal to $65 \mathrm{~mm}$ and $51 \mathrm{~mm}$. The material is SSD, certified as UNS S31803 F51 by the manufacturer (UGITECH, France). The specimens were fabricated with a slightly reduced maximum diameter (nominal value: $D_{\mathrm{e}}+10 \mathrm{~mm}$ ) to allow for a small clearance of $0.2 \mathrm{~mm}$ in the holes of the supporting plates. The rolled bars were supplied in the solution annealed condition because the material yield strength was greater than $450 \mathrm{MPa}$. This type of stainless steel is much stronger (i.e. twice or more) than the common austenitic stainless steel.

\section{Material tests}

Before testing the SSPs, three uniaxial tensile tests were performed on round coupon specimens designed according to EN10002-1 (European Committee for Standardization, 2001). The coupon specimens had an external diameter of $16 \mathrm{~mm}$ and were tapered to a reduced diameter of $12 \mathrm{~mm}$. Table 1 lists the mechanical properties of the material from the coupon tests, i.e. the yield stress $f_{\mathrm{y}}$ defined using the $0.2 \%$ offset strain, the ultimate (peak) 
stress $f_{\mathrm{u}}$, the fracture strain $\varepsilon_{\mathrm{f}}$, and the Young's modulus $E$. The average yield stress is equal to $520 \mathrm{MPa}$, the average ultimate stress is equal to $750 \mathrm{MPa}$, and the average fracture strain is 0.47 , which indicate a material with large fracture capacity and high post-yield stiffness. The ratio of the post-yield stiffness to the elastic stiffness is equal 1/125.

\section{Testing apparatus}

Tests on SSPs were conducted using a self-reacting structural testing machine employing a servo-hydraulic actuator with $2000 \mathrm{kN}$ force capacity and $\pm 120 \mathrm{~mm}$ stroke capacity. The test setup had a configuration that reproduces the SSP-gusset plate connection of the dual CBFMRF. Fig. 3 shows the test setup, which consists of vertical steel plates representing the gusset plate tied to the beam-column connection and the U-shaped plate tied to the bracing member, respectively [see Fig. 1(b)]. The SSPs were inserted into aligned holes drilled on the vertical plates. The top row of holes was used for the SSP1, whereas the bottom row was used for the SSP2. The top assembly is made of a 40-mm thick vertical plate welded normally onto a 50-mm thick 300x200 $\mathrm{mm}$ horizontal plate. The bottom assembly is made of two vertical 60-mm thick plates welded normally onto a 700x150x50 mm horizontal plate. Two 150x300x50 mm plates welded onto the top and bottom horizontal plates are gripped by the testing machine, as shown in Fig. 3. The minimum thickness of the supporting plates is based on the design rules presented by Vasdravellis et al. (2014).

Fig. 4 shows the SSP1 specimen installed. To prevent the unidirectional axial translation due to cyclic loading observed in Vasdravellis et al. (2014), the SSPs were axially restrained by welding a 10-mm thick steel collar at both ends of an SSP as shown in Fig. 4. Before the test, the collar was just in contact with the vertical plates. To prevent excessive bending of the vertical plates of the bottom assembly as the SSP deforms, $30 \mathrm{~mm}$-thick triangular stiffeners were welded at the base of the plates.

\section{Instrumentation}


Fig. 4 shows the two linear variable differential transformers (LVDTs) that were used to measure the relative displacement between the top and bottom plate assemblies. The LVDTs have $\pm 150 \mathrm{~mm}$ travel length and were fixed to the bottom horizontal plate by magnetic bases, while their tips were attached to the top horizontal plate.

\section{Loading protocols}

Table 2 lists the loading protocols that were used for the tests. All the loading protocols were applied under displacement control at a rate ranging from 5 to $40 \mathrm{~mm} / \mathrm{min}$. The first loading protocol, denoted as AISC protocol, is the one recommended in ANSI/AISC 341-10 (AISC 2010) for the seismic evaluation of buckling restrained braces. The loading history is defined by the yield displacement of the SSP, $u_{\mathrm{y}}$, and the displacement demand in the brace expected under the DBE, $u_{\mathrm{DBE}}$. The values of $u_{\mathrm{DBE}}$ were determined from the seismic evaluation results in Baiguera et al. (2016). Preliminary values of $u_{\mathrm{y}}$ were derived from the results of the simulations using the three-dimensional FEM sub-models of the SSPs presented in Baiguera et al. (2016). Based on the above, $u_{\mathrm{y}}$ is equal to $8 \mathrm{~mm}$ and $u_{\mathrm{DBE}}$ equal to $17 \mathrm{~mm}$ for SSP1, while the same quantities are equal to $5 \mathrm{~mm}$ and $14 \mathrm{~mm}$ for SSP2. The AISC protocol prescribes a loading history that consists of increasing imposed displacements with amplitudes $u_{\mathrm{y}}, 0.5 u_{\mathrm{DBE}}, u_{\mathrm{DBE}}, 1.5 u_{\mathrm{DBE}}$, and $2 u_{\mathrm{DBE}}$, each one applied for two cycles. To fully characterize the hysteretic response of each SSP up to fracture, the AISC protocol was extended to include four additional cycles at $1.5 u_{\mathrm{DBE}}$, followed by two cycles at $2.5 u_{\mathrm{DBE}}$, and then a series of cycles with an amplitude increased by $0.5 u_{\mathrm{DBE}}$ every two cycles. Both specimens were tested under ultra-low cycle fatigue loading histories, i.e. constant amplitude (CA) and randomly-generated protocols. The imposed amplitudes are defined as multiples of the SSP yield displacement. SSP1 was tested under CA $=4 u_{\mathrm{y}}, 5 u_{\mathrm{y}}, 6 u_{\mathrm{y}}$ and $7 u_{\mathrm{y}}$, while SSP2 was tested under $\mathrm{CA}=4 u_{\mathrm{y}}, 5 u_{\mathrm{y}}, 6 u_{\mathrm{y}}, 7 u_{\mathrm{y}}$ and $8 u_{\mathrm{y}}$. Both specimens were also tested under random loading protocols, which consisted of randomly generated number of 
cycles and imposed displacements. These protocols were defined assuming imposed displacement values in the range of 2 to 8 times $u_{\mathrm{y}}$ and number of cycles between 1 and 9 . Note that the selected range of applied displacements for the tests reflects the demand that SSPs are expected to resist in the proposed dual CBF-MRF, where larger displacements lead to the activation of the friction pads.

\section{EXPERIMENTAL RESULTS}

\section{Cyclic behavior and fracture of SSPs}

Fig. 5 shows the force-displacement cyclic behavior of the two specimens under the extended AISC loading protocol. $u_{\mathrm{DBE}}$ is shown on the graphs as a vertical line. The SSPs successfully passed the imposed protocol showing stable hysteretic behavior up to an imposed displacement equal to $4.5 u_{\mathrm{DBE}}$, where the tests were terminated as no signs of fracture initiation in the SSPs were observed.

The rest of the tests were executed up to full-section fracture of the specimens. Fig. 6 shows the hysteresis of SSP1 and SSP2 under the CA loading protocols. Table 2 reports the number of cycles sustained by each specimen until full-section fracture. The SSPs sustained many inelastic cycles before fracture, showing a stable hysteretic behavior and large energy dissipation capacity.

Fracture typically initiated on the surface of the SSP at the middle sections of the bending parts, i.e. halfway between $D_{\mathrm{e}}$ and $D_{\mathrm{i}}$, as shown in Fig. 7(a). These fracture locations are denoted as sections 1 and 2, where section 1 is the one closest to the lower supporting plate. The number of cycles to fracture initiation were recorded for each ultra-low cycle fatigue test and are reported in Table 2. Once fracture initiation occurred, several micro-cracks were gradually formed and propagated to full section fracture after several cycles [Fig. 7(b)]. Fig. 7(c) shows the cracks observed on the surface after forty-eight cycles in the SSP2 tested under $\mathrm{CA}=6 u_{\mathrm{y}}$ and how they propagated in the successive eleven cycles, leading to the full- 
section fracture of the specimen at cycle fifty-nine. The optimized shape of the SSPs resulted in large plastic deformations throughout the length of their bending parts. This caused a large axial elongation of the SSPs which increased with cycles. Fig. 8 shows the noticeable axial elongation of SSP2 after thirty cycles under CA $=7 u_{\mathrm{y}}$.

The force-displacement curves are characterized by a slight pinching at zero force due to the small clearance $(0.2 \mathrm{~mm})$ in the holes of the supporting plates that allows the pins to slip. It can be observed from Figs. 5 and 6 that the hysteretic curves exhibit a hardening behavior at large imposed displacement. This behavior is more evident in the CA protocols for CA> $6 u_{\mathrm{y}}$ for SSP1 and CA> $5 u_{\mathrm{y}}$ for SSP2. This hardening response is attributed to the welded collars that at large imposed displacements bore on the vertical plates, while they were not in contact with them at small amplitudes (as shown in Fig. 8).

\section{Energy dissipation capacity}

217 The energy dissipated by a SSP in a cycle, $W$, is calculated as the area enclosed by the forcedisplacement curve. To have a consistent comparison, $W$ is normalized by the product of $u_{\mathrm{y}}$ and the corresponding yield force $F_{\mathrm{y}}$. The experimental yield forces of the two specimens are $F_{\mathrm{y}, \mathrm{SSP} 1}=150 \mathrm{kN}$ and $F_{\mathrm{y}, \mathrm{SSP} 2}=75 \mathrm{kN}$. Figs. 9(a and b) show a comparison between the energy dissipating curves of SSP1 and SSP 2 under the $7 u_{\mathrm{y}}$ and $4 u_{\mathrm{y}}$ CA loading protocols. The energy dissipation capacity of SSP1 and SSP2 is similar during the first cycles, with SSP2 experiencing a more visible drop in its energy dissipation capacity than SSP1. However, SSP2 sustained a larger number of cycles than SSP1. appears to have a higher energy dissipation capacity in the initial cycles. This observation is consistent to all tests and can be attributed to the fact that the clearance between the external diameter and the supporting plate holes was slightly bigger in SSP2 than in SSP1. However, 
SSP2 reached full-section fracture after having sustained more cycles than SSP1 under CA loading protocols.

Fig. 10 compares the energy dissipation capacity of the SSPs under all the CA loading protocols. The energy dissipation curves are descending until fracture with a rate that is proportional to the magnitude of the imposed displacement, i.e. the larger the $\mathrm{CA}$ is, the faster the energy dissipation capacity of the SSPs degrades. On the contrary, when the specimens are subjected to small amplitudes (i.e. $\mathrm{CA}=4 u_{\mathrm{y}}$ ), the energy dissipation curve is almost horizontal until fracture.

\section{Prediction of strength of SSPs}

The strength of an SSP is predicted using the design equations presented in Vasdravellis et al. (2014) with modifications to account for the exact location of the plastic hinges. Fig. 7(a) shows that the plastic hinges form at midway between $D_{\mathrm{e}}$ and $D_{\mathrm{i}}$. Therefore, the strength of an SSP is given by Vasdravellis et al. (2014):

$$
F_{\mathrm{SSP}}=\frac{2}{3} \frac{D_{\mathrm{PH}}^{3}}{L_{\mathrm{PH}}} f_{\mathrm{y}}
$$

where $L_{\mathrm{PH}}=L_{\mathrm{SSP}} / 2$, and $D_{\mathrm{PH}}=\left(D_{\mathrm{e}}+D_{\mathrm{i}}\right) / 2$, based on the geometric properties shown in Fig. 2 . Using this formula, the strength of SSP1 is $156 \mathrm{kN}$ and that of SSP2 is $75 \mathrm{kN}$, which are in excellent agreement with the experimental values, i.e. $150 \mathrm{kN}$ and $75 \mathrm{kN}$, respectively. Note that the capacity design rules to avoid shear failure at the section of diameter $D_{\mathrm{i}}$ are satisfied according to Vasdravellis et al. (2014).

\section{PREDICTION OF FRACTURE OF SSPS USING THE PALMGREN-}

\section{MINER'S RULE}

The results of the CA tests were used to derive a relationship between the applied displacement amplitude and the number of cycles to fracture. Such correlation may be convenient for establishing a fracture criterion in phenomenological models of the SSPs for 
seismic collapse modeling of buildings equipped with such dampers. For instance, the 'Fatigue material' model available in the OpenSEES software (Mazzoni et al. 2006), which is based on the Coffin-Manson relationship and on a linear damage accumulation rule, can also be defined for spring-like elements with a force-displacement response.

Based on the CA test results, the points corresponding to the number of cycles to fracture $\left(N_{\mathrm{f}}\right)$ as a function of the applied amplitude $\left(\Delta_{\mathrm{f}} / 2\right)$ are plotted in Fig. 11. Then, a Coffin-Mansonlike equation can be obtained, i.e.

$$
\Delta_{\mathrm{f}} / 2=\Delta_{0} \cdot\left(N_{\mathrm{f}}\right)^{m}
$$

where $m$ and $\Delta_{0}$ are parameters with values that result in the best fit to the points in Fig. 11 . The calibrated values of $\Delta_{0}$ and $m$ are $350 \mathrm{~mm}$ and -0.6 for SSP1, and $455 \mathrm{~mm}$ and -0.6 for SSP2.

The Palmgren-Miner linear damage accumulation rule is applied to the random tests:

$$
D=\sum_{i=1}^{j} \frac{n_{\mathrm{i}}}{N_{\mathrm{f}, \mathrm{i}}}
$$

where $n_{\mathrm{i}}$ is the number of cycles applied at a given amplitude, $N_{\mathrm{f}, \mathrm{i}}$ is the number of cycles required to reach fracture at that given amplitude, and $D$ is the damage index, which is equal to 1 when the low-cycle fatigue life is reached (Bruneau et al. 2011). Table 3 shows that the fracture prediction using Eq. (3) for the randomly generated cyclic loading protocols is in good correlation with the experimental results: SSP1 fractured at the end of phases 14 and 9 for which the Miner's rule estimates a value of $D$ equal to 1.14 and 1.08 , respectively, while SSP2 fractured at the end of phase 9 for which the Miner's rule estimates a value of $D$ equal to 0.99 .

The calibrated parameters are only valid for the specific geometries tested in this study. Instead, the mechanics-based fracture models, presented below, can be used to estimate the facture behavior of new geometries under ULCF, without the need for further tests. 


\section{MICROMECHANICS-BASED FRACTURE MODELS}

\section{Fracture prediction under monotonic loading}

276 Under monotonic loading, the Void Growth Model (VGM) and the Stress Modified Critical

277 Strain (SMCS) model provide good predictions of ductile fracture in metals based on prior theoretical and experimental research (McClintock 1968; Rice and Tracey 1969; Hancock and Mackenzie 1976; Mackenzie et al. 1977; Hancock and Brown 1983; Johnson and Cook 1985; Marini et al. 1985; Panontin and Sheppard 1995; Bandstra et al. 2004; Anderson 2005; Kanvinde and Deierlein 2006; Kanvinde 2017). These studies have shown that ductile fracture depends on two variables, i.e. the equivalent plastic strain $\bar{\varepsilon}^{p l}$ and the stress triaxiality, which is defined as the ratio of the mean stress, $\sigma_{\mathrm{m}}$, to the von Mises stress, $\sigma_{\mathrm{e}}$.

The VGM assumes that ductile fracture initiates when a quantity named void growth index (VGI $\left.I_{\text {monotonic }}\right)$ reaches a critical value $\left(\mathrm{VGI}_{\text {monotonic }}^{\text {critical }}\right)$ :

$$
\mathrm{VGI}_{\text {monotonic }}=\int_{0}^{\bar{\varepsilon}^{p l}} \exp (1.5 T) \mathrm{d} \bar{\varepsilon}^{p l}>\mathrm{VGI}_{\text {monotonic }}^{\text {critical }}
$$

286 Calculation of the $\mathrm{VGI}_{\text {monotonic }}^{\text {critical }}$, which is considered as a material property invariant to stress and strain states, requires complementary FEM analysis up to the point of fracture initiation (Kanvinde and Deierlein 2006).

The SMCS model does not account for variations in triaxiality during the loading history.

Fracture initiation occurs when $\bar{\varepsilon}^{p l}$ reaches the critical value $\bar{\varepsilon}_{\text {critical }}^{p l}$ :

$$
\bar{\varepsilon}_{\text {critical }}^{p l}=\alpha \exp (-1.5 T)
$$

where $\alpha$ is the toughness index. The SMCS model requires complementary FEM analysis to

292 calibrate $\alpha$, based on $\bar{\varepsilon}_{\text {critical }}^{p l}$ and $T$ values at fracture initiation. The SMCS model was recently applied to predict fracture in various steel grades and in steel beam-column connections (Chi et al. 2006; Kanvinde and Deierlein 2006). Kiran and Khandelwal (2013) calibrated the parameters of the VGM and SMCS models for the A992 steel grade. 
296 The Abaqus software offers a general criterion for predicting ductile fracture initiation that is

297 given by:

$$
\omega_{\text {critical }}=\int \frac{\mathrm{d} \bar{\varepsilon}^{p l}}{\bar{\varepsilon}_{\text {critical }}^{p l}(T)}
$$

298

299

300

301

302

303

304

305

306

307

308

309

310

311

312

313

314

315

where $\omega_{\text {critical }}$ is the fracture initiation index that increases monotonically with plastic deformations and $\bar{\varepsilon}_{\text {critical }}^{p l}(T)$ is the equivalent plastic strain at fracture initiation, which depends on the instantaneous $T$ value (Dassault Systèmes 2014). When $\omega_{\text {critical }}=1$, it is assumed that fracture initiation occurs.

More recently, a fracture criterion under monotonic loading that depends on both the triaxiality and the Lode angle parameter was proposed in Wen and Mahmoud (2016a).

\section{Fracture prediction under ultra-low cycle fatigue}

In seismic applications, the initiation of ductile fracture in metals typically occurs due to ULCF, i.e. the material is subjected to a relatively small number of large inelastic cycles. Under this loading condition, the fracture mechanism is more similar to monotonic ductile fracture rather than low or high cycle fatigue failure that typically involves hundreds or thousands of cycles. To predict fracture initiation in metals under ULCF, Kanvinde and Deierlein (2007) proposed the CVGM, which is an extension of the VGM accounting for positive and negative triaxiality that develops at the point of interest under cyclic loading:

$$
\mathrm{VGI}_{\text {cyclic }}=\int_{T \geq 0} \exp (1.5|T|) \mathrm{d} \bar{\varepsilon}^{p l}-\int_{T<0} \exp (1.5|T|) \mathrm{d} \bar{\varepsilon}^{p l}
$$

The model assumes that fracture initiates in the material only under positive triaxiality.

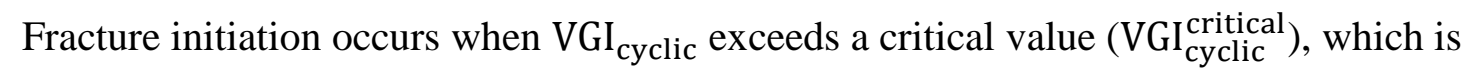
calculated applying an exponential decay function to its monotonic critical value VGI $\mathrm{Imonotonic}_{\text {critical }}$, i.e.

$$
\mathrm{VGI}_{\text {cyclic }}^{\text {critical }}=\mathrm{VGI}_{\text {monotonic }}^{\text {critical }} \exp \left(-\lambda \bar{\varepsilon}_{\text {acc }}^{p l}\right)
$$


316

317

where $\bar{\varepsilon}_{\text {acc }}^{p l}$ is the cumulative plastic strain up to the start of each tensile excursion and $\lambda$ is the rate of cyclic deterioration, which takes values from 0 to 1 for structural steels (Kanvinde and Deierlein 2007). A small value of $\lambda$ results in a faster degradation. The coefficient $\lambda$ is experimentally determined by conducting cyclic tests on CNSs.

Jia and Kuwamura (2015) have recently simulated ductile fracture of specimens subjected to cyclic loading using the Abaqus fracture initiation criterion [Eq. (6)]. To define an $\bar{\varepsilon}_{\text {critical }}^{p l}(T)$ function appropriate for cyclic loading, they modified the SMCS model by introducing a cutoff at $T=-1 / 3$, on the basis of experimental evidence that ductile fracture is practically inhibited in compression (Bridgman 1964; Bao \& Wierzbicki 2004). Below $T=-1 / 3$, ductile fracture is assumed to initiate for an infinite value of $\bar{\varepsilon}_{\text {critical }}^{p l}(T)$ and thus no damage is accumulated. The above conditions are expressed as:

$$
\begin{gathered}
\bar{\varepsilon}_{\text {critical }}^{p l}(T)= \begin{cases}\alpha_{\text {cyclic }} \exp (-1.5 T) & \text { if } T \geq-1 / 3 \\
\infty & \text { if } T<-1 / 3\end{cases} \\
\omega_{\text {critical }}= \begin{cases}\int \frac{\mathrm{d} \bar{\varepsilon}^{p l}}{\bar{\varepsilon}_{\text {critical }}^{p l}(T)} & \text { if } T \geq-1 / 3 \\
0 & \text { if } T<-1 / 3\end{cases}
\end{gathered}
$$

This fracture criterion was previously validated by Jia \& Kuwamura (2015) against the response of specimens monotonically pulled to fracture after being subjected to few small inelastic cycles (fewer than five). For this purpose, the cyclic fracture parameter $\alpha_{\text {cyclic }}$ was calibrated using monotonic tests on round specimens. However, its application to ultra-low cycle fatigue requires the calibration of $\alpha_{\text {cyclic }}$ based on coupon tests under cyclic loading. All models derived from the work of McClintock (1968) and Rice and Tracey (1969) assume that the stress state is axisymmetric. However, recent studies have demonstrated that ductile fracture is also influenced by the Lode angle $\theta$, which is an additional indicator of stress state and related to the Lode parameter, $\xi$, as expressed in Eq. (11): 


$$
\xi=\cos \theta=\frac{3 \sqrt{3}}{2} \frac{J_{3}}{J_{2}^{3 / 2}}
$$

where $J_{2}$ and $J_{3}$ are the second and third stress invariants of the deviatoric stress tensor. $\xi$ varies from -1 , in case of axisymmetric compression, to 1 , in case of axisymmetric tension. Smith et al. (2014) and Smith et al. (2017) recently proposed the stress-weighted damage model (SWDM), which is an enhanced version of the CVGM accounting for the effect of the deviatoric stress state. Wen and Mahmoud (2016b) developed a new fracture model that takes in full consideration both stress triaxiality and the Lode angle parameter.

In this study, the effect of the Lode angle parameter is not considered since complementary FEM simulations of the SSP tests show that the fracture locations are characterized by axisymmetric stress state, i.e. $\xi= \pm 1$ [see Fig. 23(c)] at the locations of fracture on the SSPs' external surfaces. This indicates that the deviatoric stress does not influence the prediction of ductile fracture initiation in the SSPs under ULCF.

\section{CALIBRATION OF FRACTURE PARAMETERS FOR DUPLEX}

\section{STAINLESS STEEL}

\section{CNS tests}

Monotonic and cyclic tests on CNSs made of duplex stainless steel were carried out to calibrate the critical parameters of the CVGM and the Abaqus ductile fracture initiation model. CNSs with three different radii were used, i.e. $R=2,3$, ad $4.5 \mathrm{~mm}$, to vary the severity of triaxiality at the center of the notched cross-section. The notched specimens, denoted as CNS-2, CNS-3, and CNS-4.5, were manufactured using 16-mm diameter round bars from the same material batch of the SSPs. The CNS geometries are shown in Fig. 12(a). CNS-2 is characterized by high triaxiality $(T>1)$ at the center of the notch, while CNS-3 and CNS-4.5 have moderate triaxiality $(1 / 3<T<1)$. 
358 A total of six tests, three tensile monotonic and three cyclic, were conducted for each CNS up to fracture. Two types of ultra-low cycle fatigue protocols were defined, i.e. CA, consisting of cycles between zero and a positive displacement multiple of the yield displacement $d_{\mathrm{y}}$; and protocols with increasing amplitude where the specimen was subjected to amplitudes increased by $2 d_{\mathrm{y}}$ every four cycles. Table 4 provides a summary of the loading protocols. The specimens were instrumented with a 50-mm gauge length extensometer as shown in Fig. 12(b). The tests were performed under displacement control with a rate of $1 \mathrm{~mm} / \mathrm{min}$. The imposed displacement was controlled by the extensometer.

The monotonic force-displacement curves of CNS-2 are shown in Fig. 13(a). Fig. 13(b) shows the cyclic force-displacement response of CNS-4.5 under no. 9 protocol. The CNSs showed a stable hysteretic response under all cyclic protocols. Ductile fracture of the specimen occurred in all the tests.

\section{FEM simulations of the coupon tests}

Nonlinear three-dimensional FEM models of the CNSs were created in Abaqus. Fig. 12(b) shows the geometry of the FEM model of CNS-2. Only the gauge length was modelled and was discretized using C3D8R elements with reduced integration. The mesh is refined in the notch with an average element size of $0.45 \mathrm{~mm}$. The displacement history measured by the extensometer was applied defining a smooth step amplitude. The model was analyzed using the explicit dynamic solver in Abaqus as the explicit direct integration procedure is computationally efficient for the simulation of highly discontinuous quasi-static problems that involve contact, damage and failure. To reduce the computational cost of quasi-static simulations, a smaller loading rate is typically applied. In addition, a variable mass scaling is used for computational efficiency by defining a minimum stable time increment target. 
smooth step amplitude was defined for the FEM simulations of the monotonic tests to ensure a stable quasi-static analysis.

An elastic plastic constitutive law with isotropic hardening, shown in Fig. 14, was specified for the monotonic tests, based on coupon tests on round bars performed prior to the CNS tests. To capture the cyclic behavior of duplex stainless steel, an elastic plastic material model with combined isotropic and kinematic hardening was specified. The material model is defined by the yield surface $\varphi(\sigma)$ defined as (Dassault Systemes, 2016):

$$
\varphi(\boldsymbol{\sigma})=\sqrt{\frac{3}{2}(\boldsymbol{S}-\boldsymbol{\alpha})^{t}(\boldsymbol{S}-\boldsymbol{\alpha})}-\sigma^{0}
$$

390

where $\sigma^{0}$ is the yield stress, $t$ is the transposition operation, $S$ is the stress deviator, $\sigma$ is the stress vector and $\alpha$ is the backstress vector. The hardening laws for each backstress are defined as:

$$
\begin{gathered}
\boldsymbol{\alpha}=\sum_{\mathrm{k}=1}^{\mathrm{B}} \boldsymbol{\alpha}_{\mathrm{k}} \\
\dot{\boldsymbol{\alpha}}_{\mathrm{k}}=\frac{C_{\mathrm{k}}}{\sigma^{0}}(\boldsymbol{\sigma}-\boldsymbol{\alpha}) \dot{\bar{\varepsilon}}^{p}-\gamma_{\mathrm{k}} \boldsymbol{\alpha}_{\mathrm{k}} \dot{\bar{\varepsilon}}^{p}
\end{gathered}
$$

where a superimposed dot indicates an incremental quantity, $B$ is the total number of the backstresses, $C_{\mathrm{k}}$ and $\gamma_{\mathrm{k}}$ are the constitutive material parameters to be calibrated against the experimental results, and $\dot{\bar{\varepsilon}}^{p}$ is the equivalent plastic strain rate. The evolution of $\sigma^{0}$ (isotropic hardening component) is defined by the following exponential law:

$$
\sigma^{0}=\left.\sigma\right|_{0}+Q_{\infty}\left(1-e^{-b \bar{\varepsilon}^{p}}\right)
$$

where $\left.\sigma\right|_{0}$ is the yield stress at zero plastic strain, $b$ defines the rate at which the size of $\varphi(\sigma)$ changes for increasing plastic strains, and $Q_{\infty}$ is the maximum change in the size of $\varphi(\sigma)$.

Several simulations were iteratively conducted to identify the values of the parameters that define the constitutive model. A good correlation was achieved adopting the following values: $\left.\sigma\right|_{0}=400 \mathrm{MPa}, C_{1}=6,500 \mathrm{MPa}, \gamma_{1}=30, C_{2}=100,000 \mathrm{MPa}, \gamma_{2}=700, b=5, Q_{\infty}=$ 
$200 \mathrm{MPa}$. Fig. 13 shows the experimental-numerical agreement for CNS-2 under monotonic loading and CNS-4.5 under no. 9 cyclic protocol. A similar agreement was found in all the tests.

\section{Calibration of the CVGM}

The parameters of the CVGM, i.e. VGI monotonic and $\lambda$, were calibrated following the procedure described in Kanvinde \& Deierlein (2007). First, the VGI monotonic was identified based on the FEM simulations of the monotonic CNS tests. Then, the cyclic damage parameter $\lambda$ was identified using the FEM simulations of the cyclic CNS tests.

Stress and strain histories extracted from the fracture location of the CNSs, i.e. the center of the notched section, were used to integrate Eq. (4) up to fracture (assumed to represent complete failure of the specimen) as indicated in the force-displacement response of CNS-2 in Fig. 13(a). The values of VGI monotonic along with the plastic strain and triaxiality at fracture for the three CNS geometries under monotonic loading are summarized in Table 5. VGI $I_{\text {monotonic }}^{\text {critical }}$ has a mean value of 2.88 and a small standard deviation equal to 0.29 . This value agrees with the results presented in Vasdravellis et al. (2014), where VGI monotonic was found to have a mean value of 2.87 for the SSD material.

The value of the parameter $\lambda$ was determined by deriving a relationship between the $\mathrm{VGI}_{\text {cyclic }}^{\text {critical }} / \mathrm{VGI}_{\text {monotonic }}^{\text {critical }}$ ratio and the associated $\bar{\varepsilon}_{\text {acc }}^{p l}$ at fracture initiation. Damage initiation in the cyclic tests is assumed to occur when there is a $10 \%$ drop in the force carrying capacity of the specimen based on the force time history. Fig. 15(a) shows the force versus cycle evolution for CNS-2 subjected to no. 3 loading protocol (Table 4). Fracture initiation is indicated on the graph by the vertical shaded area and the cycle where fracture initiated is denoted as $N_{0}$, i.e. $N_{0}=18$ in this test. VGI cyclic critical values are calculated by integrating Eq. (7) for each cyclic CNS test. By fitting an exponential function to the resulting VGI cyclic / 
426 VGI monotonic $_{\text {critical }}-\bar{\varepsilon}_{\text {acc }}^{p l}$ data, plotted in Fig. $16, \lambda=0.12$. The small value of $\lambda$ obtained for SSD

427 is consistent with the large fracture capacity exhibited by the coupon specimens.

\section{Calibration of the ductile fracture initiation and evolution criterion in Abaqus}

The calibration of the Abaqus ductile fracture initiation criterion involves determining the parameter $\alpha_{\text {cyclic }}$ in Eq. (9) based on the cyclic CNS test results. Thus, $\alpha_{\text {cyclic }}$ is calibrated using the same stress and strain histories extracted from the FEM simulations for the calibration of the CVGM. The fracture initiation index $\omega_{\text {critical }}$ is determined by integrating Eq. (10) and $\alpha_{\text {cyclic }}$ is iteratively found imposing $\omega_{\text {critical }}=1$ at the start of the cycle where fracture initiated. Fig. 15(b) shows the evolution of $\omega_{\text {critical }}$ in the test no. 3 of CNS-2. To have $\omega_{\text {critical }}=1$ at the $18^{\text {th }}$ cycle, $\alpha_{\text {cyclic }}$ should be equal to 10 in this test. The same procedure of determining $\alpha_{\text {cyclic }}$ was applied to all CNS cyclic tests and the results are summarized in Table

6. $\alpha_{\text {cyclic }}$ has a mean value of 10.6 and a standard deviation of 1.4. The excessively small value of 5.5 resulted for specimen CNS-2 under no. 2 loading protocol was disregarded as non-representative. Note that the specimen in the specific test sustained fewer cycles than in test no. 3 despite being subjected to a smaller amplitude. Thus, $\alpha_{\text {cyclic }}=10$ is conservatively used in the fracture simulations of the SSPs in Abaqus. Fig. 17 shows the $\bar{\varepsilon}_{\text {critical }}^{p l}(T)$ function expressed in Eq. (9) with $\alpha_{\text {cyclic }}=10$ and the cut-off at $T=-1 / 3$.

To simulate the progressive degradation of the material following fracture initiation, Abaqus offers a damage evolution criterion based on the approach proposed by Hillerborg et al. (1976). The stress-strain definition cannot accurately capture the degradation of the material as a strain localization would introduce a strong mesh dependency. Abaqus overcomes this issue by introducing a damaged stress-displacement response (Dassault Systèmes 2014). The damage evolution variable is specified as a function of the equivalent plastic displacement $\bar{u}^{p l}$. The latter depends on the characteristic length of a finite element $L_{\mathrm{char}}$, which is expressed by: 


$$
\bar{u}^{p l}=L_{\text {char }} \dot{\varepsilon}^{p l}
$$

451 Before fracture initiation, $\bar{u}^{\dot{p}}=0$. Since $L_{\text {char }}$ depends on the geometry and formulation of

452 the finite element, the mesh dependency of the results is reduced (Dassault Systèmes 2014).

453 In addition, the damage evolution capability offers the removal of the elements from the

454 mesh when the damage evolution index $D_{\text {evol }}$ in Eq. (17) is equal to 1:

$$
D_{\text {evol }}=1-\frac{\sigma_{\mathrm{dam}}}{\sigma}
$$

455 where $\sigma_{\text {dam }}$ is the 'damaged' stress of the material (Dassault Systèmes 2014). The

456 calibration procedure proposed in Pavlovic et al. (2013) was used in this study to define the

457 damage evolution law. The characteristic length of a finite element is given by the product of the element size and a factor accounting for the element type (e.g. 3.2 for C3D8R elements in Abaqus). The evolution of the damage variable $D_{\text {evol, }}$ specified as a tabular function of $\bar{u}^{p l}$, was derived using the results of tensile coupon tests on round bars. Details of this calibration procedure can be found in Pavlovic et al. (2013) and are not repeated herein.

\section{Validation of fracture parameters using the CNS tests}

To validate the Abaqus fracture models for ultra-low cycle fatigue loading, the cyclic CNS tests were simulated in Abaqus/Explicit using the fracture parameters described in the previous section. The experimental and numerical hysteresis and force evolutions of three cyclic tests (one for each CNS geometry) are shown in Fig. 18. The calibrated Abaqus fracture initiation and evolution model capture well the response of CNSs.

\section{Three-dimensional FEM models of SSP tests}

471 Three-dimensional FEM models of the full-scale tests on SSPs were constructed in Abaqus.

472 Only half of the test setup was reproduced in full detail due to its symmetric geometry. The 
steel collar and the triangular stiffeners were included in the model. Fig. 19 shows the mesh discretization applied to the FEM model of SSP1 along with the boundary conditions. Threedimensional hexahedral elements with reduced integration (C3D8R) were used for all the parts of the assembly. A symmetry condition was defined to the nodes of the symmetry plane. The grip of the testing machine jaw faces was simulated by restraining all the degrees of freedom on the surface of the vertical plate welded to the bottom plate assembly. The imposed displacement history was applied to the upper supporting plate assembly as shown in Fig. 19. A relatively coarse mesh was used for the steel plate assemblies, while a more refined mesh is applied to the SSPs, where inelastic deformations and fracture were experimentally observed. To keep the computational time of analysis at reasonable levels, the average mesh size in the bending parts of the SSPs was $3 \mathrm{~mm}$. It is noted that unlike fracture in existing crack tips or sudden geometric changes, the stress state at the free surface of a SSP is smooth, and the fracture models are less sensitive to the mesh size (Vasdravellis et al. 2014). Therefore, the adopted mesh was considered a reasonable trade-off between computational time and accuracy.

Surface-based tie constraints, which impose equal displacements among the nodes of two surfaces, were used for modelling the welded joints in the two steel plate assemblies, i.e. between the lower and vertical plates, the triangular stiffeners at the base of the plates, and the steel washer welded onto the SSP. The welds around the supporting plates (Fig. 3) were not included in the FEM model because preliminary analyses showed that their effect is negligible. A general contact algorithm was defined to simulate the interaction between the SSP and the holes of the supporting plates. Based on experimental measurements, a clearance of $0.1 \mathrm{~mm}$ and $0.3 \mathrm{~mm}$ was used for the SSP1 and SSP2 models, respectively. A contact property with normal and tangential behavior with a friction coefficient equal to 0.2 was

497 defined between the SSP and the holes of the supporting plates. 
The hysteretic behavior of duplex stainless steel was simulated by the elastic-plastic material model with combined isotropic and kinematic hardening. An elastic-plastic material model with isotropic hardening behavior was defined for the steel assemblies made of S355 grade steel. The yield stress of S355 steel was conservatively reduced to $300 \mathrm{MPa}$ to account for the large thickness of the steel plates $(40-60 \mathrm{~mm})$ since the yield stress reduces with increasing thickness of plate sections (European Committee for Standardization 2004).

\section{Explicit FEM simulations without fracture}

To evaluate the ability of the FEM model to capture the cyclic hardening of the SSPs and to adjust the various parameters of the explicit solver so that it can capture the quasi-static loading conditions, the cyclic tests were first simulated in Abaqus/Explicit without the definition of any ductile fracture criteria. Displacement-controlled analyses were conducted under quasi-static loading conditions in the large displacement/strain nonlinear regime. To ensure that that the loading rate is relatively low and no dynamic effects influence the analysis, the time step for one cycle was set equal to $60 \mathrm{sec}$. For example, for an imposed amplitude of $49 \mathrm{~mm}$, the load was applied at around $3 \mathrm{~mm} / \mathrm{s}$. To ensure a stable analysis, the density of the material was decreased by six orders of magnitude, and the displacement history was applied with a periodic amplitude. Based on the mesh size, a stable target time increment equal to $0.0001 \mathrm{sec}$ was iteratively identified.

Fig. 20 shows the comparison of the numerical and experimental hysteresis of SSP1 under $\mathrm{CA}=7 u_{\mathrm{y}}$ and SSP2 under $\mathrm{CA}=6 u_{\mathrm{y}}$. The results indicate that the FEM model is capable of tracing well the cyclic behavior of the specimens prior to fracture. Similar correlations are found for the rest of the loading protocols. It can be observed that the FEM simulations capture the pinching effect at zero force, indicating that the clearance between the SSPs and the holes of the supporting plates was modelled accurately.

\section{CVGM fracture predictions}


523 The SSP simulations were post-processed to evaluate the accuracy of the CVGM to predict

524 fracture in the SSPs. The stress and strain histories at the locations of fracture, i.e. at mid-

525 distance between $D_{\mathrm{e}}$ and $D_{\mathrm{i}}$ (Fig. 3), were extracted at the end of the analyses. The results

526 were then used to derive the $\mathrm{VGI}_{\text {cyclic }}$ and $\mathrm{VGI}_{\text {cyclic }}^{\text {critical }}$ histories.

527 Fig. 21 shows the evolutions of $\mathrm{VGI}_{\text {cyclic }}$ and $\mathrm{VGI}_{\text {cyclic }}^{\text {critical }}$ for the $\mathrm{CA}=6 u_{\mathrm{y}}$ test of SSP2.

$528 \mathrm{VGI}_{\text {cyclic }}$ varies with the sign of $T$, while $\mathrm{VGI}_{\text {cyclic }}^{\text {critical }}$ is a stepwise function starting at

529 VGI monotonic and decreasing at the start of each cycle according to the exponential decay

530 function given by Eq. (8). The intersection of the $\mathrm{VGI}_{\text {cyclic }}^{\text {critical }}$ and $\mathrm{VGI}_{\text {cyclic }}$ curves indicates

531 fracture. As illustrated in Fig. 21, the CVGM predicts fracture at the same cycle observed in

532 the test. The CVGM fracture predictions are summarized in Table 7 for all tests. The results

533 indicate that the calibrated CVGM parameters predict with good accuracy the fracture in the

534 SSPs with a maximum error of $12 \%$.

535 Explicit simulation of SSP fracture in Abaqus

536 Explicit fracture simulations of the SSPs were performed in Abaqus using the fracture

537 initiation criterion shown in Fig. 16. The parameters of the damage evolution model, which

538 depends on the mesh size (i.e. $L_{\text {char }}$ ), were modified to account for the 3-mm average element

539 size used in the bending parts of the SSPs.

540 Fig. 22 shows a comparison between the experimental and numerical deformed shapes at the onset of fracture initiation for both SSPs under CA $=7 u_{\mathrm{y}}$. The contours of the fracture initiation index, i.e. the output variable DUCTCRT, are plotted on the numerical models. When DUCTRT $=1$, then fracture has initiated in the model at the corresponding location. It

544 is shown that the FEM simulations predict the exact location of fracture in the SSPs, i.e. at locations 1 and 2, which are midway between $D_{\mathrm{e}}$ and $D_{\mathrm{i}}$.

546 The evolution of the variables governing ductile fracture, extracted at the location of fracture

547 from the simulation of SSP2 under the random protocol, are shown in Fig. 23. In Fig. 23(a), 
the evolution of the damage variable $\omega_{\text {critical }}$ during the cyclic loading is plotted. It takes the value 1 at the beginning of the $41^{\text {st }}$ cycle, indicating fracture initiation. After this point, degradation initiates according to the specified damage evolution law until the element removal from the mesh. The histories of both $\omega_{\text {critical }}$ and $T$ over three consecutive cycles of the simulation, i.e. cycles 25 to 28, are plotted in Fig. 23(b). It is shown that triaxiality at the fracture section is characterized by alternating cycles of tension and compression with maximum absolute values in the range of $0.33-0.4$. It can also be observed that, below the cut-off value of $T=-1 / 3$, no damage is accumulated. It is noted that the Lode parameter $\xi$ at fracture initiation is in within 0.96-1 [Fig. 23(c)]. This indicates that the fracture location in a SSP under cyclic loading is characterized by axisymmetric stress state and therefore the effect of the Lode angle is negligible on the prediction of ductile fracture.

The results of fracture initiation predictions for all the simulations are summarized in Table 8, where the cycle at which fracture initiates is compared with that from the experiments. The predictions are within $\pm 10 \%$ error. The latter has a mean value of $6 \%$ and standard deviation of $1.2 \%$. Thus, it can be concluded that the calibrated model in Abaqus/Explicit can provide an accurate prediction for all the ultra-low cycle fatigue tests. Following fracture initiation, the numerical force-carrying capacity of the SSPs decreases because of the deletion of elements from the mesh according to the damage evolution model. Fig. 24 compares the simulated fracture evolution with experimental photographic evidence of two representative cyclic tests on SSP1 and SSP2 (no. 2 and 11 tests in Table 2). The results show that the FEM model can simulate the progressive damage of the material due to cyclic loading after fracture initiation until complete fracture of the section occurs. However, comparison of the numerical and experimental force histories of the same tests in Fig. 25 reveals that, once fracture initiates, the numerical force-carrying capacity decreases at a faster rate than in the experiments. A similar response can be seen in the numerical-experimental 
573 force evolutions of the remaining tests. This indicates that the FEM simulation tends to

574 underestimate the numbers of cycles between fracture initiation and complete failure. For

575 instance, simulations of $\mathrm{CA}=4 u_{\mathrm{y}}$ tests show a premature degradation of the force-carrying

576 capacity of SSPs. Such discrepancy can be attributed to the relatively coarse mesh applied to

577 the SSP bending parts. For an improved accuracy in simulating fracture evolution, a refined

578 mesh should be ideally used at fracture locations. However, this would result in a significant

579 increase in computational time.

\section{CONCLUSIONS}

581 This paper presented an experimental and numerical investigation on the cyclic behavior and

582 facture capacity of SSPs under ULCF conditions. SSPs are devices with large post-yield

583 stiffness ratio, which can be used in series with conventional steel braces to increase the

584 energy dissipation capacity and reduce the residual drifts of steel frames. The tests conducted

585 on SSPs included fourteen ultra-low cycle fatigue loading protocols. Three predictive ductile

586 fracture models were calibrated and assessed against the test results. Based on the findings of

587 this work, the following conclusions can be drawn:

588 - SSPs successfully pass the AISC loading protocol, sustaining without fracture

589 displacements up to 4.5 times the displacement demand of the Design Basis Earthquake.

590 - Under constant amplitude cyclic protocols, SSPs sustain many inelastic cycles without

591 degradation before initiation of ductile fracture.

592 - The optimized shape of the SSPs results in large plastic deformations throughout the

593 whole length of the bending parts. Ductile fracture initiates at the free surface, at a section

594 half way between the maximum and minimum diameter.

595 - The Palmgren-Miner's rule predicts failure of SSPs under the randomly generated

596 loading protocols with very good accuracy, and thus, the calibrated Coffin-Manson-like relationships can be reliably applied to phenomenological fracture models for seismic 
collapse analysis of buildings equipped with these devices. However, the parameters associated with this rule depend on the geometry of the SSPs examined in this study.

- $\quad$ The calibrated micromechanics-based models, i.e. the CVGM and the built-in Abaqus criterion calibrated for cyclic loading, provide accurate predictions of ductile fracture initiation for the ULCF tests of SSPs. The Cyclic Void Growth Model (CVGM) predicts ductile fracture in SSPs under all loading protocols with a maximum error of $12 \%$, mean error of $6 \%$, and standard deviation of $5 \%$, while the Abaqus model predicts fracture initiation with maximum error of $9 \%$, mean error of $4 \%$, and standard deviation of $3 \%$. Therefore, the calibrated fracture parameters can be used to predict the ULCF fracture initiation of SSPs having different geometries and boundary conditions, without the need for further experimental tests. Note that the parameter $\alpha_{\text {cyclic }}$ in the modified Abaqus fracture model is valid only for ULCF, while the CVGM can be used for monotonic loading and ULCF.

- The Abaqus explicit fracture simulations capture well the hysteretic behavior of the SSPs; however, the ability of tracing the degradation of the material following fracture initiation was less accurate due to the relatively coarse mesh applied to the bending parts of the SSP.

\section{REFERENCES}

AISC (2010). "Seismic provisions for structural steel buildings.” ANSI/AISC 341/10, Chicago, IL.

Anderson, T. L. (2005). Fracture mechanics: Fundamentals and applications, CRC Press, Boca Raton, FL.

Baiguera, M., Vasdravellis, G., and Karavasilis, T. L. (2016). "Dual seismic-resistant steel frame with high post-yield stiffness energy-dissipative braces for residual drift reduction." $J$. Constr. Steel Res., 122, 198212. 
"Modeling void coalescence during ductile fracture of a steel." Mater. Sci. Eng. A., 366(2),

625

626

627

628

629

630

631

632

633

634

635

636

637

638

639

640

641

643

644

645

646

40

642 Dimopoulos, A. I, Karavasilis, T. L., Vasdravellis, G., and Uy, B. (2013). "Seismic design, $269-281$,

Bao, Y. and Wierzbicki, T. (2004). "On fracture locus in the equivalent strain and stress triaxiality space.” Int. J. Mech. Sci., 46(1), 81-98.

Bridgman, P. (1964). Studies in large plastic flow and fracture, Harvard University Press, Cambridge, MA.

Bruneau, M., Uang, C. M., and Sabelli, R. (2011). Ductile design of steel structures, McGraw Hill, Inc, 2011.

Chan, R. W. K., and Albermani, F. (2008). “Experimental study of steel slit damper for passive energy dissipation." Eng. Struct., 30(4), 1058-1066.

Chi, W. M., Kanvinde, A. M., and Deierlein, G. G. (2006). "Prediction of ductile fracture in welded connections using the smcs criterion.” J. Struct. Eng., 132(2), 171-181.

Ciampi, V., and Marioni, A. (1991). "New types of energy dissipating devices for seismic protection of bridges.”, Proceedings of 3rd World Congress on Joint Sealing and Bearing Systems for Concrete Structures, National Center for Earthquake Engineering Research, Buffalo, NY.

Dassault Systèmes (2014). ABAQUS documentation, 6.14 edn, Dassault Systèmes Simulia, Providence, RI. modelling and assessment of self-centering steel frames using post-tensioned connections with web hourglass shape pins.” Bull. Earthquake Eng., 11(5), 1797-1816. 
European Committee for Standardization (2004). "Hot rolled products of structural steels.

Part 2: Technical delivery conditions for non-alloy structural steels.” EN 10025-2, Brussels,

649

650

651

652

653

654

655

656

657

658

659

660

661

662

663

664

665

666

667

668

669

670

671

Belgium.

Gray, M., Christopoulos, C., and Packer, J. (2014). "Cast steel yielding brace system for concentrically braced frames: concept development and experimental validations." J. Struct. Eng., 140(4), 04013095.

Hancock, J. W., and Mackenzie, A. C. (1976). "On the mechanics of ductile failure in high-strength steel subjected to multi-axial stress states." J. Mech. Phys. Solids, 24(2-3), 147169.

Hancock, J. W., and Brown, D.K. (1983). "On the role of strain and stress state in ductile failure.” J. Mech. Phys. Solids, 31, 1-24.

Hillerborg, A., Modeer, M., and Petersson, P. E. (1976). “Analysis of crack formation and crack growth in concrete by means of fracture mechanics and finite elements." Cem. Concr. Res., 6, 773-782.

Jia, L. J., and Kuwamura, H. (2015). "Ductile fracture model for structural steel under cyclic large strain loading." J. Constr. Steel Res., 106, 110-121.

Johnson, G. B., and Cook, W. H. (1985). "Fracture characteristics of three metals subjected to various strains, strain rates, temperatures and pressures.” Eng. Fract. Mech., 21(1), 31-48.

Kajima (1991), Honeycomb Damper System, Japan, Kajima Corporation.

Kanvinde, A. M. (2017). "Predicting fracture in civil engineering steel structures: State of the art.” J. Struct. Eng., 143(3), 03116001.

Kanvinde, A. M., and Deierlein, G. G. (2006). "The void growth model and the stress modified critical strain model to predict ductile fracture in structural steels." J. Struct. Eng., 10.1061/(ASCE)0733-9445(2006), 132(12), 1907-1918. 
672

673

674

675

676

677

678

679

680

681

682

683

684

685

686

687

688

689

690

691

692

693

694

695

Kanvinde, A. M., and Deierlein, G. G. (2007). "Cyclic void growth model to assess ductile fracture initiation in structural steels due to ultra-low cycle fatigue.” J. Eng. Mech., 10.1061/(ASCE)0733-9399(2007), 133(6), 701-712.

Kelly, J. M., Skinner, R. I., and Heine, A. J. (1972). "Mechanisms of energy absorption in special devices for use in earthquake-resistant structures.” Bull. New Zealand Soc. Earthq. Eng., 5(3), 63-88.

Kiran, R., and Khandelwal, K. (2013). "Experimental studies and models for ductile fracture in ASTM A992 steels at high triaxiality." J. Struct. Eng., 140(2).

Lemaitre, J. (1985). “A continuous damage mechanics model for ductile fracture.” J. Eng. Mater. Technol., 107(1), 83-90.

Mackenzie, A. C., Hancock, J. W., and Brown, D. K. (1977). “On the influence of state of stress on ductile failure initiation in high strength steels.” Eng. Fract. Mech., 9(1), 167-188.

Marini, B., Mudry, F., and Pineau, A. (1985). "Experimental study of cavity growth in ductile rupture.” Eng. Fract. Mech., 22, 989-996.

Marioni, A. (1997). "Development of a new type of hysteretic damper for the seismic protection of bridges." Proc. $4^{\text {th }}$ World Congress on Joint Sealing and Bearing Systems for Concrete Structures, American Concrete Institute, Detroit, USA, 955-976.

Mazzoni, S., McKenna, F., Scott, M., and Fenves, G. (2006). Open System for Earthquake Engineering Simulation (OpenSees). User command language manual, Pacific Earthquake Engineering Research Center, University of California, Berkley, CA.

McClintock, F. A. (1968). "Criterion for ductile fracture by growth of holes.” J. Appl. Mech., 35(2), 363-371.

Nakashima, M., Akazawa, T., and Tsuji, B. (1995). "Strain-hardening behavior of shear panels made of low-yield steel. II: Model.” J. Struct. Eng., 121(12), 1750-1757. 

fatigue tests on structural carbon steel and stainless steel.” J. Constr. Steel Res., 66(1), 96-

698

699

700

701

702

703

704

705

706

707

708

709

710

711

712

713

714

715

716

717

718

719 110.

Oh, S. H., Kim, Y. J., and Ryu, H. S. (2009). "Seismic performance of steel structures with slit dampers" Eng. Struct., 31, 9, 1997-2008.

Panontin, T. L., and Sheppard, S. D. (1995). "The relationship between constraint and ductile fracture initiation as defined by micromechanical analyses." Proc. 26th National Symposium on Fracture Mechanics, ASTM International, Philadelphia, 54-85.

Pavlovic, M., Markovic, Z., Veljkovic, M., and Budevac, D. (2013). "Bolted shear connectors vs. headed studs behaviour in push-out tests." J. Constr. Steel Res., 88, 134-149.

Rice, J. R., and Tracey, D. M. (1969). "On the ductile enlargement of voids in triaxial stress fields." J. Mech. Phys. Solids, 17(3), 201-217.

Skinner, R. I., Kelly, J. M., and Heine, A. J. (1975). "Hysteretic dampers for earthquakeresistant structures." Earthq. Eng. Struct. Dynam., 3(3), 287-296.

Smith, C. M., Deierlein, G. G., and Kanvinde, A. M. (2014). “A stress-weighted damage model for ductile fracture initiation in structural steel under cyclic loading and generalized stress states." TR 187, Blume Earthquake Engineering Center, Stanford Univ., Stanford, CA.

Smith, C., Kanvinde, A, Deierlein, G. (2017). “A local criterion for ductile fracture under low-triaxiality axisymmetric stress states", Eng. Fract. Mech., 169, 321-335.

Soong, T. T., and Spencer, B. F. (2002). "Supplemental energy dissipation: state-of-the-art and state-of-the-practice." Eng. Struct. 24(3), 243-259.

Steimer, S. F., Godden, W. G., and Kelly, J. M. (1981). "Experimental behavior of a spatial piping system with steel energy absorbers subjected to a simulated differential seismic input.” Report UCB/EERC-81/09, Earthq. Eng. Res. Ctr, Univ. of California, Berkeley, CA. 

Johnson, M. W., and McNamara, R. J. (2008). "Energy Dissipation Systems for Seismic

722 Applications: Current Practice and Recent Developments.” J. Struct. Eng., 134(1), 3-21.

Tsai, K. C., Chen, H. W., Hong, C. P., and Su, Y. F. (1993). "Design of steel triangular plate energy absorbers for seismic-resistant construction.” Earthq. Spectra, 9(3), 505-528.

Tsopelas, P., and Constantinou, M. (1997). "Study of elastoplastic bridge seismic isolation system.”, J. Struct. Eng., 123, 4.

Vasdravellis, G., Karavasilis, T. L., and Uy, B. (2013a). “Large-scale experimental validation of steel posttensioned connections with web hourglass pins.” J. Struct. Eng., 139, $1033-1042$.

Vasdravellis, G., Karavasilis, T. L., and Uy, B. (2013b). "Finite element models and cyclic behavior of self-centering steel post-tensioned connections with web hourglass pins." Eng. Struct., 52, 1-16.

Vasdravellis, G., Karavasilis, T. L., and Uy, B. (2014). “Design rules, experimental evaluation, and fracture models for high-strength and stainless steel hourglass shape energy dissipation devices.” J. Struct. Eng., 140, 04014087.

Wen, H., and Mahmoud, H. (2016a). "New Model for Ductile Fracture of Metal Alloys. I: Monotonic Loading.” J. Eng. Mech., 142, 04015088-1.

Wen, H., and Mahmoud, H. (2016b). "New Model for Ductile Fracture of Metal Alloys. II: Reverse Loading.” J. Eng. Mech., 142, 04015089-1.

Whittaker, A. S., Bertero, V. V., Thompson, C. L., and Alonso, L. J. (1991). "Seismic testing of steel plate energy dissipation devices.” Earthq. Spectra, 7(4), 563-604. 
745

746

747

748

749 
TABLE 1. Summary of mechanical properties of duplex stainless steel

\begin{tabular}{lcccc}
\hline \hline Specimen & $\begin{array}{c}f_{\mathrm{y}} \\
(\mathrm{MPa})\end{array}$ & $\begin{array}{c}f_{\mathrm{u}} \\
(\mathrm{MPa})\end{array}$ & $\begin{array}{c}e_{\mathrm{f}} \\
(\%)\end{array}$ & $\begin{array}{c}E \\
(\mathrm{MPa})\end{array}$ \\
\hline Round bar 1 & 530 & 752.4 & 45.7 & 189,655 \\
Round bar 1 & 513 & 750.9 & 47.5 & 181,250 \\
Round bar 1 & 518 & 745.8 & 47.9 & 187,500 \\
Mean & 520 & 749.7 & 47.0 & 186,135 \\
\hline \hline
\end{tabular}


TABLE 2. Test matrix of SSP tests

\begin{tabular}{|c|c|c|c|c|c|}
\hline \multirow[t]{2}{*}{ Specimen } & \multirow[t]{2}{*}{ Test } & \multirow[t]{2}{*}{ Protocol } & \multirow[t]{2}{*}{ Failure mode } & \multicolumn{2}{|c|}{ No. of cycles } \\
\hline & & & & Full fracture & Fracture initiation \\
\hline \multirow[t]{7}{*}{ SSP1 } & 1 & AISC & No failure & - & - \\
\hline & 2 & $\mathrm{CA}=7 u_{\mathrm{y}}$ & Ductile fracture & 28 & 21 \\
\hline & 3 & $\mathrm{CA}=6 u_{\mathrm{y}}$ & Ductile fracture & 35 & 25 \\
\hline & 4 & $\mathrm{CA}=5 u_{\mathrm{y}}$ & Ductile fracture & 44 & 31 \\
\hline & 5 & $\mathrm{CA}=4 u_{\mathrm{y}}$ & Ductile fracture & 78 & 43 \\
\hline & 6 & Random-1 & Ductile fracture & 59 & 35 \\
\hline & 7 & Random-2 & Ductile fracture & 45 & 25 \\
\hline \multirow[t]{7}{*}{ SSP2 } & 8 & AISC & No failure & - & - \\
\hline & 9 & $\mathrm{CA}=8 u_{\mathrm{y}}$ & Ductile fracture & 33 & 30 \\
\hline & 10 & $\mathrm{CA}=7 u_{\mathrm{y}}$ & Ductile fracture & 43 & 36 \\
\hline & 11 & $\mathrm{CA}=6 u_{\mathrm{y}}$ & Ductile fracture & 59 & 41 \\
\hline & 12 & $\mathrm{CA}=5 u_{\mathrm{y}}$ & Ductile fracture & 76 & 45 \\
\hline & 13 & $\mathrm{CA}=4 u_{\mathrm{y}}$ & Ductile fracture & 89 & 54 \\
\hline & 14 & Random & Ductile fracture & 48 & 40 \\
\hline
\end{tabular}


TABLE 3. Prediction of fracture in SSPs under random loading protocols using the Palmgren-Miner rule

\begin{tabular}{|c|c|c|c|c|c|c|}
\hline Specimen & Test & Phase & $\Delta_{\mathrm{f}} / 2$ & $n$ & $N_{\mathrm{f}}$ & $D$ \\
\hline \multirow[t]{14}{*}{ SSP1 } & Random-1 & 1 & $3 u_{\mathrm{y}}$ & 8 & 109 & 0.07 \\
\hline & & 2 & $6 u_{\mathrm{y}}$ & 1 & 35 & 0.10 \\
\hline & & 3 & $4 u_{\mathrm{y}}$ & 9 & 78 & 0.22 \\
\hline & & 4 & $7 u_{\mathrm{y}}$ & 2 & 28 & 0.29 \\
\hline & & 5 & $6 u_{\mathrm{y}}$ & 5 & 35 & 0.43 \\
\hline & & 6 & $5 u_{\mathrm{y}}$ & 3 & 44 & 0.50 \\
\hline & & 7 & $3 u_{\mathrm{y}}$ & 4 & 109 & 0.54 \\
\hline & & 8 & $7 u_{\mathrm{y}}$ & 7 & 28 & 0.79 \\
\hline & & 9 & $3 u_{\mathrm{y}}$ & 2 & 109 & 0.81 \\
\hline & & 10 & $7 u_{\mathrm{y}}$ & 3 & 28 & 0.91 \\
\hline & & 11 & $3 u_{\mathrm{y}}$ & 2 & 109 & 0.93 \\
\hline & & 12 & $4 u_{\mathrm{y}}$ & 2 & 78 & 0.96 \\
\hline & & 13 & $2 u_{\mathrm{y}}$ & 4 & 214 & 0.98 \\
\hline & & 14 & $5 u_{\mathrm{y}}$ & $7^{\mathrm{a}}$ & 44 & 1.14 \\
\hline \multirow[t]{9}{*}{ SSP1 } & Random-2 & 1 & $6 u_{\mathrm{y}}$ & 9 & 35 & 0.26 \\
\hline & & 2 & $7 u_{\mathrm{y}}$ & 8 & 28 & 0.54 \\
\hline & & 3 & $2 u_{\mathrm{y}}$ & 2 & 214 & 0.55 \\
\hline & & 4 & $7 u_{\mathrm{y}}$ & 6 & 28 & 0.77 \\
\hline & & 5 & $5 u_{\mathrm{y}}$ & 4 & 44 & 0.86 \\
\hline & & 6 & $2 u_{\mathrm{y}}$ & 6 & 214 & 0.89 \\
\hline & & 7 & $3 u_{\mathrm{y}}$ & 4 & 109 & 0.92 \\
\hline & & 8 & $5 u_{\mathrm{y}}$ & 5 & 44 & 1.04 \\
\hline & & 9 & $7 u_{\mathrm{y}}$ & $1^{\mathrm{a}}$ & 28 & 1.08 \\
\hline \multirow[t]{9}{*}{ SSP2 } & Random & 1 & $6 u_{\mathrm{y}}$ & 9 & 59 & 0.15 \\
\hline & & 2 & $7 u_{\mathrm{y}}$ & 8 & 43 & 0.34 \\
\hline & & 3 & $2 u_{\mathrm{y}}$ & 2 & 33 & 0.40 \\
\hline & & 4 & $7 u_{\mathrm{y}}$ & 6 & 43 & 0.54 \\
\hline & & 5 & $5 u_{\mathrm{y}}$ & 4 & 76 & 0.59 \\
\hline & & 6 & $2 u_{\mathrm{y}}$ & 6 & 33 & 0.77 \\
\hline & & 7 & $3 u_{\mathrm{y}}$ & 4 & 168 & 0.80 \\
\hline & & 8 & $5 u_{\mathrm{y}}$ & 5 & 76 & 0.86 \\
\hline & & 9 & $7 u_{\mathrm{y}}$ & $5^{\mathrm{a}}$ & 43 & 0.99 \\
\hline
\end{tabular}

${ }^{a}$ Experimental fracture 
TABLE 4. Cyclic loading protocols of CNS tests

\begin{tabular}{ccc}
\hline \hline Specimen & Test & Loading protocol \\
\hline CNS-2 & 1 & $(4) \mathrm{x}\left[0 ; 4 d_{\mathrm{y}}\right]+(4) \mathrm{x}\left[0 ; 6 d_{\mathrm{y}}\right]+(2) \mathrm{x}\left[0 ; 8 d_{\mathrm{y}}\right]+\mathrm{p} . \mathrm{t} . \mathrm{f}$. \\
& 2 & $(24) \mathrm{x}\left[0 ; 5 d_{\mathrm{y}}\right]$ \\
& 3 & $(24) \mathrm{x}\left[0 ; 6 d_{\mathrm{y}}\right]$ \\
CNS-3 & 4 & $(4) \mathrm{x}\left[0 ; 4 d_{\mathrm{y}}\right]+(4) \mathrm{x}\left[0 ; 6 d_{\mathrm{y}}\right]+(4) \mathrm{x}\left[0 ; 8 d_{\mathrm{y}}\right]+(4) \mathrm{x}\left[0 ; 10 d_{\mathrm{y}}\right]+(1) \mathrm{x}\left[0 ; 12 d_{\mathrm{y}}\right]$ \\
& 5 & $(39) \mathrm{x}\left[0 ; 5 d_{\mathrm{y}}\right]$ \\
& 6 & $(41) \mathrm{x}\left[0 ; 5 d_{\mathrm{y}}\right]+\mathrm{p} . \mathrm{t} . \mathrm{f}$. \\
$\mathrm{CNS}-4.5$ & 7 & $(4) \mathrm{x}\left[0 ; 4 d_{\mathrm{y}}\right]+(4) \mathrm{x}\left[0 ; 6 d_{\mathrm{y}}\right]+(4) \mathrm{x}\left[0 ; 8 d_{\mathrm{y}}\right]+(4) \mathrm{x}\left[0 ; 10 d_{\mathrm{y}}\right]+(2) \mathrm{x}\left[0 ; 12 d_{\mathrm{y}}\right]$ \\
& 8 & $(19) \mathrm{x}\left[0 ; 8 d_{\mathrm{y}}\right]$ \\
& 9 &
\end{tabular}

Note: the number in parentheses indicates the number of cycles, followed by the prescribed amplitude in square brackets. For example, $(22) x\left[0 ; 5 d_{\mathrm{y}}\right]$ refers to a specimen subjected to twenty-two cycles between 0 and 5 times $d_{\mathrm{y}}$; p.t.f. $=$ pull to fracture. 
TABLE 5. Summary of VGI monotonic $_{\text {critical }}^{\text {Tand }} \bar{\varepsilon}^{p l}$ values at fracture for CNS tests

\begin{tabular}{|c|c|c|c|c|}
\hline Specimen & & VGI monotonic & $T^{*}$ & $\bar{\varepsilon}^{p l *}$ \\
\hline CNS-2 & & 2.66 & 1.02 & 0.77 \\
\hline CNS-3 & & 3.21 & 0.76 & 1.00 \\
\hline \multirow[t]{3}{*}{ CNS-4.5 } & & 2.77 & 0.63 & 1.08 \\
\hline & Mean & 2.88 & & \\
\hline & St dev & 0.29 & & \\
\hline
\end{tabular}

*Note: the values of $T$ and $\bar{\varepsilon}^{p l}$ refer to the monotonic coupon tests. 
TABLE 6. Summary of $\alpha_{\text {cyclic }}$ values for the CNS tests

\begin{tabular}{|c|c|c|c|}
\hline Specimen & Cyclic Test & $N_{0}$ & $\alpha_{\text {cyclic }}$ \\
\hline \multirow[t]{3}{*}{ CNS-2 } & 1 & p.t.f. & 9.0 \\
\hline & 2 & 16 & $5.5^{\mathrm{a}}$ \\
\hline & 3 & 18 & 10.0 \\
\hline \multirow[t]{3}{*}{ CNS-3 } & 4 & 16 & 11.2 \\
\hline & 5 & 16 & 13.4 \\
\hline & 6 & 32 & 9.9 \\
\hline \multirow[t]{5}{*}{ CNS-4.5 } & 7 & p.t.f. & 11.6 \\
\hline & 8 & 18 & 9.5 \\
\hline & 9 & 18 & 10.2 \\
\hline & & & 10.6 \\
\hline & & & 1.2 \\
\hline
\end{tabular}

${ }^{a}$ Value ignored as not representative 
TABLE 7. Prediction of fracture initiation in SSPs according to CVGM versus experimental tests

\begin{tabular}{|c|c|c|c|c|c|c|}
\hline \multirow[t]{3}{*}{ Specimen } & \multirow[t]{3}{*}{ Test } & \multirow[t]{3}{*}{ Protocol } & \multicolumn{4}{|c|}{ Fracture initiation $N_{0}$} \\
\hline & & & \multirow{2}{*}{$\begin{array}{c}\text { Test } \\
\text { (cycle no.) }\end{array}$} & \multirow{2}{*}{$\begin{array}{c}\text { CVGM } \\
\text { (cycle no.) }\end{array}$} & \multicolumn{2}{|c|}{ CVGM-test difference } \\
\hline & & & & & (cycle) & (\% error) \\
\hline \multirow[t]{6}{*}{ SSP1 } & 2 & $\mathrm{CA}=7 u_{\mathrm{y}}$ & 21 & 19 & -2 & $-10 \%$ \\
\hline & 3 & $\mathrm{CA}=6 u_{\mathrm{y}}$ & 25 & 28 & +3 & $+12 \%$ \\
\hline & 4 & $\mathrm{CA}=5 u_{\mathrm{y}}$ & 31 & 29 & -2 & $-6 \%$ \\
\hline & 5 & $\mathrm{CA}=4 u_{\mathrm{y}}$ & 43 & 42 & -1 & $-2 \%$ \\
\hline & 6 & Random-1 & 35 & 35 & 0 & $0 \%$ \\
\hline & 7 & Random-2 & 25 & 28 & +3 & $+12 \%$ \\
\hline \multirow[t]{8}{*}{ SSP2 } & 9 & $\mathrm{CA}=8 u_{\mathrm{y}}$ & 30 & 33 & +3 & $+10 \%$ \\
\hline & 10 & $\mathrm{CA}=7 u_{\mathrm{y}}$ & 36 & 36 & 0 & $0 \%$ \\
\hline & 11 & $\mathrm{CA}=6 u_{\mathrm{y}}$ & 41 & 41 & 0 & $0 \%$ \\
\hline & 12 & $\mathrm{CA}=5 u_{\mathrm{y}}$ & 45 & 45 & 0 & $0 \%$ \\
\hline & 13 & $\mathrm{CA}=4 u_{\mathrm{y}}$ & 54 & 48 & -6 & $-11 \%$ \\
\hline & 14 & Random & 40 & 42 & +2 & $+5 \%$ \\
\hline & & & & & Mean & $6 \%$ \\
\hline & & & & & St dev & $5 \%$ \\
\hline
\end{tabular}


TABLE 8. Prediction of fracture initiation in SSPs according to Abaqus fracture model versus experimental tests

\begin{tabular}{|c|c|c|c|c|c|c|}
\hline \multirow[t]{3}{*}{ Specimen } & \multirow[t]{3}{*}{ Test } & \multirow[t]{3}{*}{ Protocol } & \multicolumn{4}{|c|}{ Fracture initiation $N_{0}$} \\
\hline & & & \multirow{2}{*}{$\begin{array}{c}\text { Test } \\
\text { (cycle no.) }\end{array}$} & \multirow{2}{*}{$\begin{array}{c}\text { Abaqus } \\
\text { (cycle no.) }\end{array}$} & \multicolumn{2}{|c|}{ Abaqus-test difference } \\
\hline & & & & & (cycle) & (\% error) \\
\hline \multirow[t]{6}{*}{ SSP1 } & 2 & $\mathrm{CA}=7 u_{\mathrm{y}}$ & 21 & 21 & 0 & $0 \%$ \\
\hline & 3 & $\mathrm{CA}=6 u_{\mathrm{y}}$ & 25 & 26 & +1 & $+4 \%$ \\
\hline & 4 & $\mathrm{CA}=5 u_{\mathrm{y}}$ & 31 & 29 & -2 & $-6 \%$ \\
\hline & 5 & $\mathrm{CA}=4 u_{\mathrm{y}}$ & 43 & 40 & -3 & $-7 \%$ \\
\hline & 6 & Random-1 & 35 & 36 & +1 & $+3 \%$ \\
\hline & 7 & Random-2 & 25 & 26 & +1 & $+4 \%$ \\
\hline \multirow[t]{8}{*}{ SSP2 } & 9 & $\mathrm{CA}=8 u_{\mathrm{y}}$ & 30 & 32 & +2 & $+7 \%$ \\
\hline & 10 & $\mathrm{CA}=7 u_{\mathrm{y}}$ & 36 & 37 & +1 & $+3 \%$ \\
\hline & 11 & $\mathrm{CA}=6 u_{\mathrm{y}}$ & 41 & 42 & +1 & $+2 \%$ \\
\hline & 12 & $\mathrm{CA}=5 u_{\mathrm{y}}$ & 45 & 45 & 0 & $0 \%$ \\
\hline & 13 & $\mathrm{CA}=4 u_{\mathrm{y}}$ & 54 & 49 & -5 & $-9 \%$ \\
\hline & 14 & Random & 40 & 41 & +1 & $+3 \%$ \\
\hline & & & & & Mean & $4 \%$ \\
\hline & & & & & St dev & $3 \%$ \\
\hline
\end{tabular}




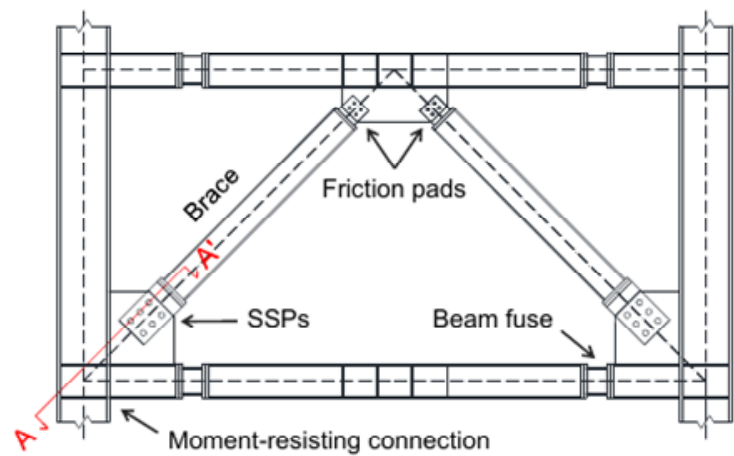

(a)

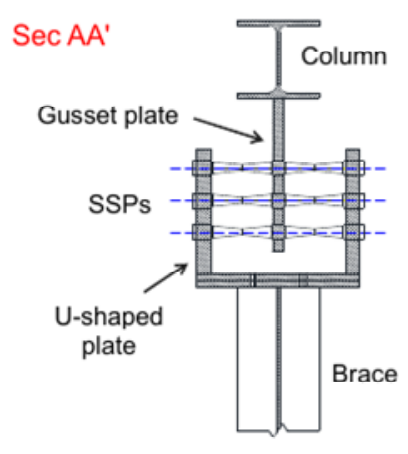

(b)

Fig. 1. Geometry of the dual CBF-MRF proposed in Baiguera et al. (2016): (a) overview; and (b) brace-SSP connection detail 


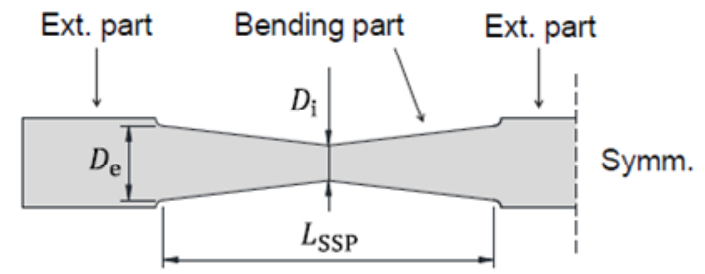

(a)

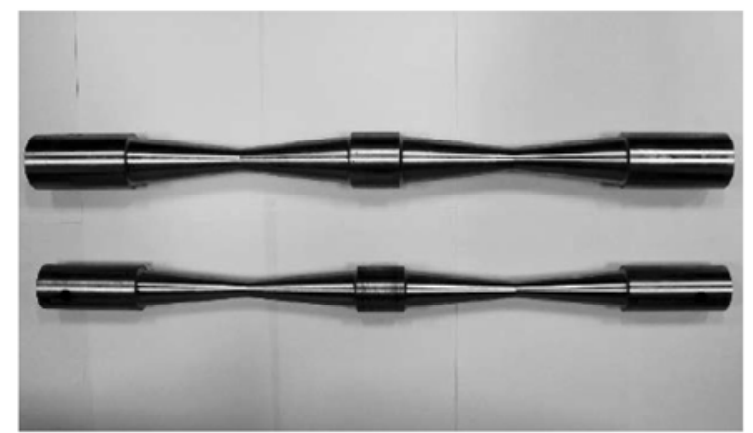

(b)

Fig. 2. (a) SSP geometry; and (b) SSP specimens 


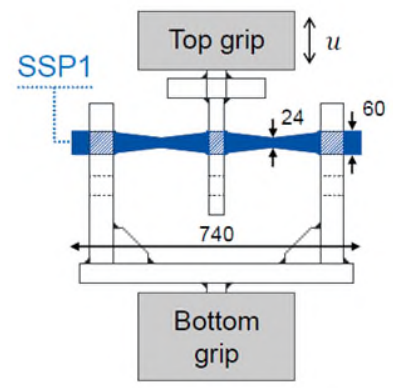

(a)

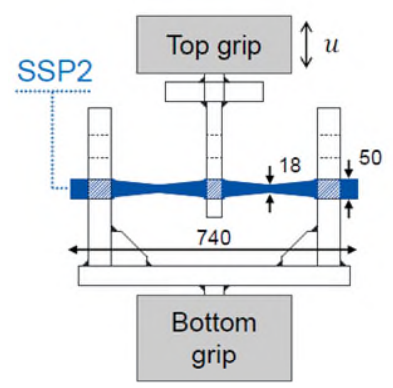

(b)

Fig. 3. Test setup: (a) SSP1; and (b) SSP2 


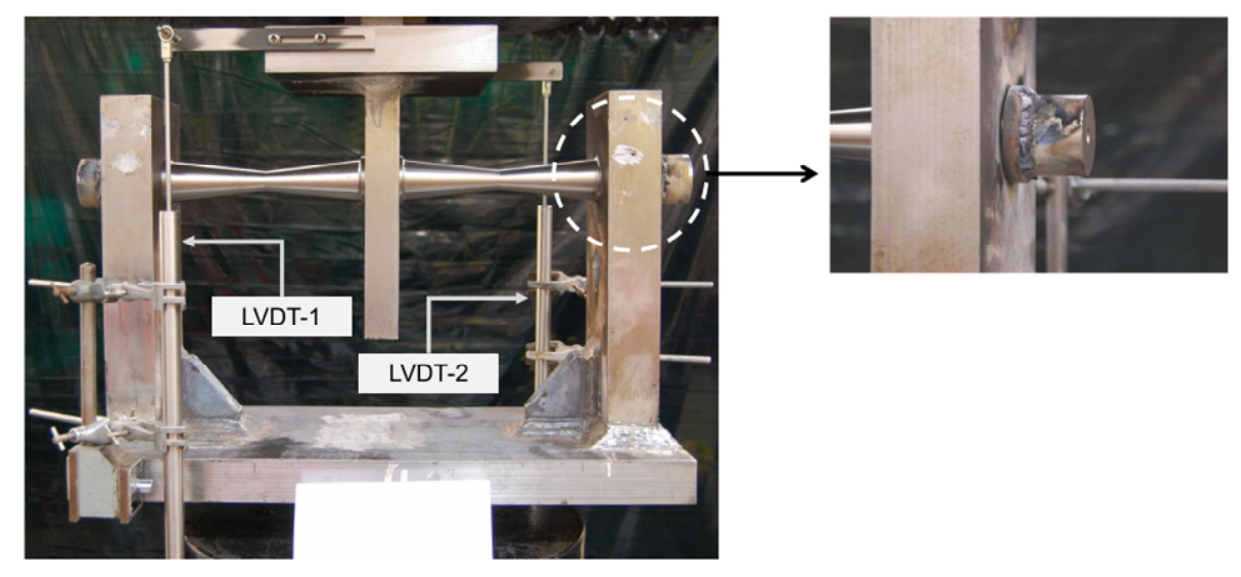

Fig. 4. Test setup and welded collar on SSP1 

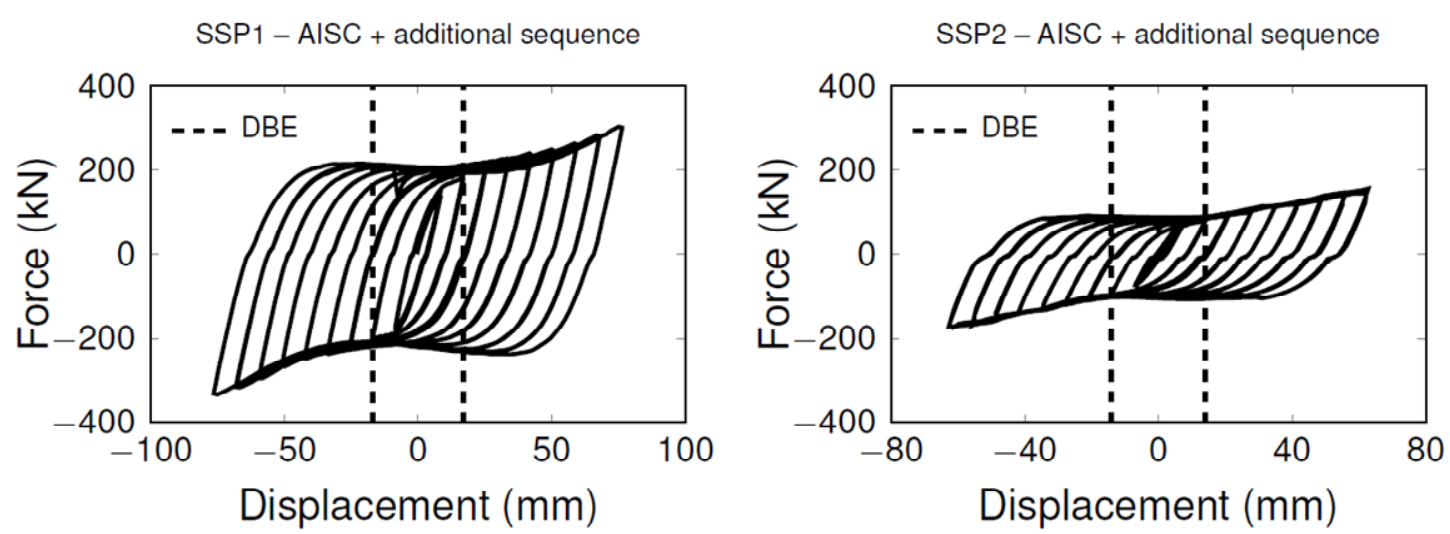

Fig. 5. Hysteresis of SSPs under the AISC protocol with additional cycles up to four and half times $u_{\mathrm{DBE}}$ 

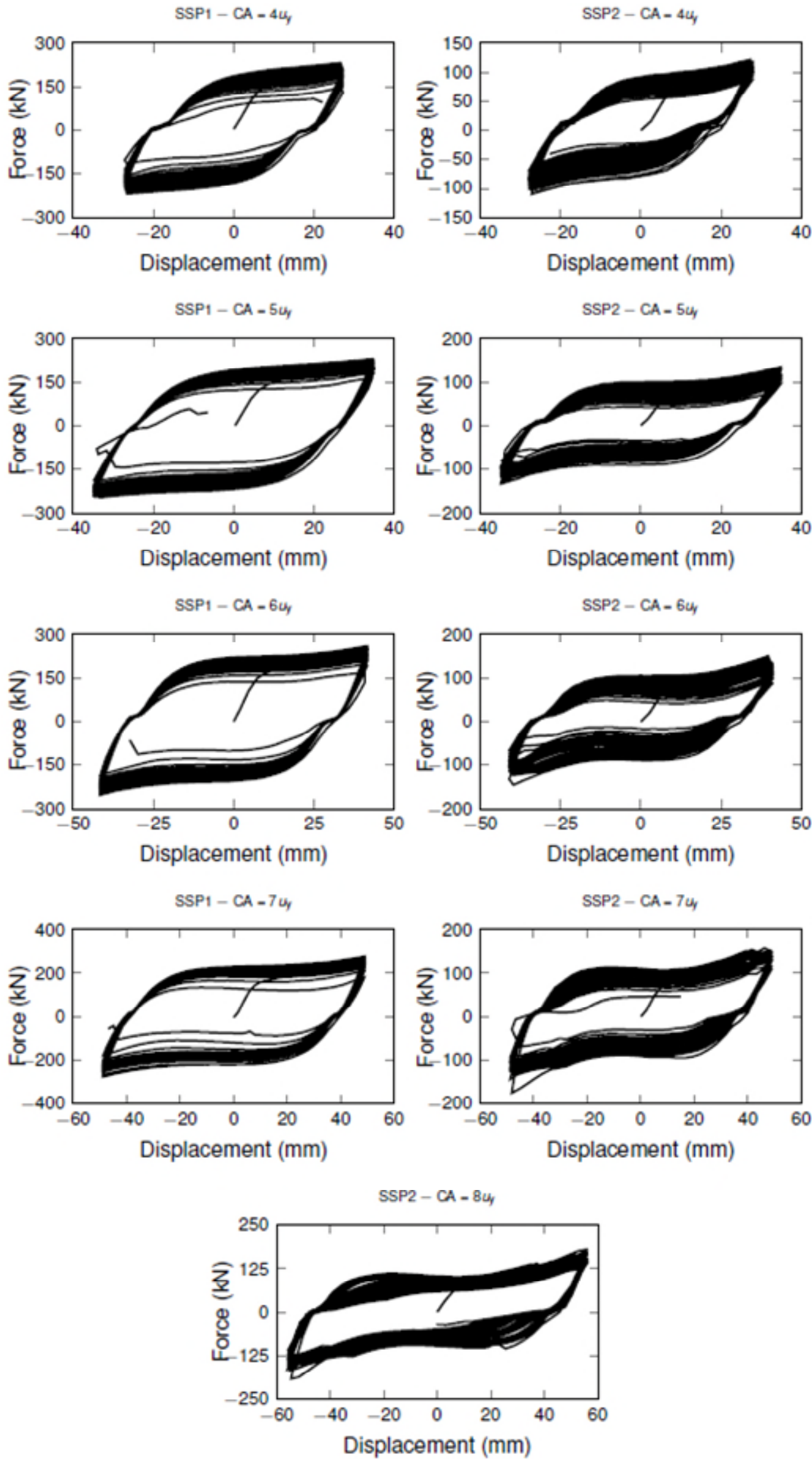

Fig. 6. Hysteresis of SSPs under the CA protocols 


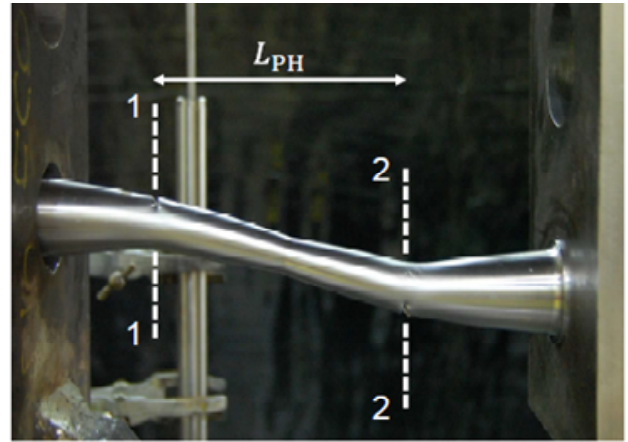

(a)

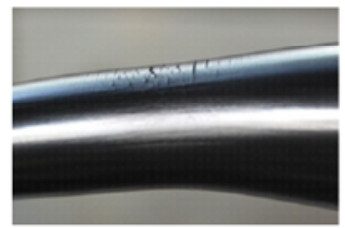

Cycle no. 48

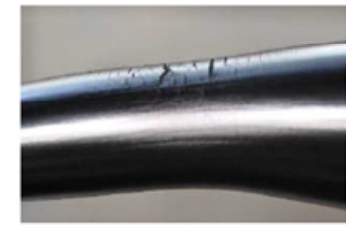

Cycle no. 51

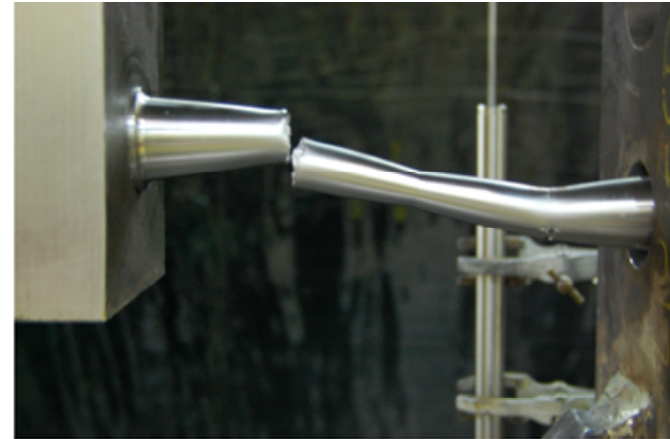

(b)

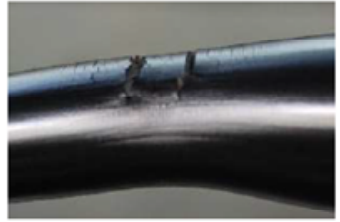

Cycle no. 54

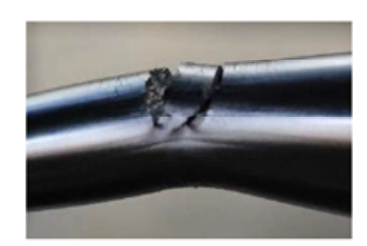

Cycle no. 59 (final)

(c)

Fig. 7. (a) Typical fracture locations in SSPs; (b) full section fracture; and (c) fracture evolution in SSP2 under CA $=6 u_{\mathrm{y}}$ 


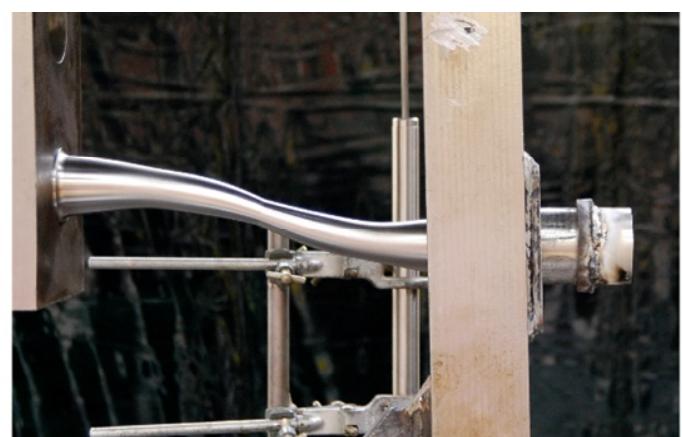

Fig. 8. Axial elongation of SSP 2 after 30 cycles under $\mathrm{CA}=7 u_{\mathrm{y}}$ 


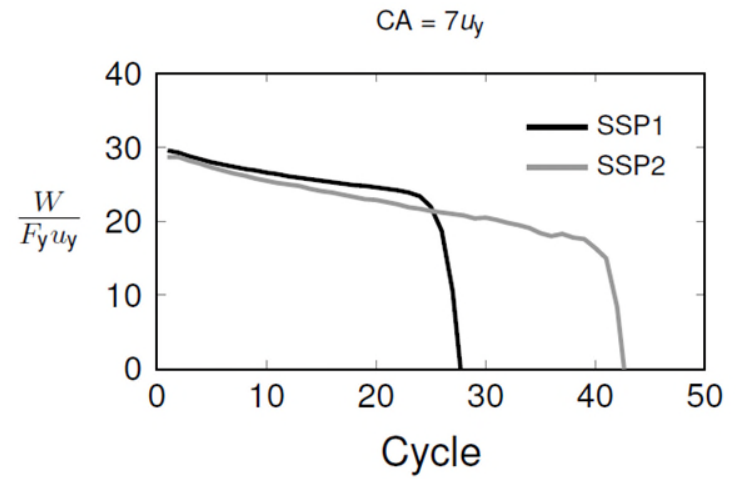

(a)

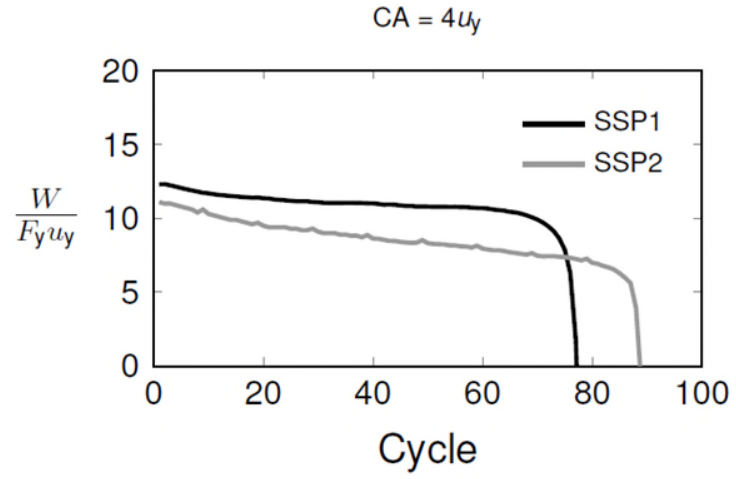

(b)

AISC

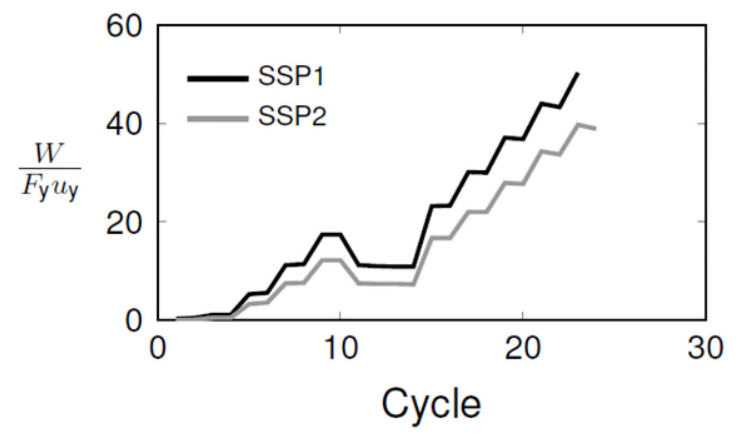

(c)

Fig. 9. Comparison of the energy dissipation of SSPs: (a) $\mathrm{CA}=7 u_{\mathrm{y}}$ test; (b) $\mathrm{CA}=4 u_{\mathrm{y}}$ test; and (c) AISC test 


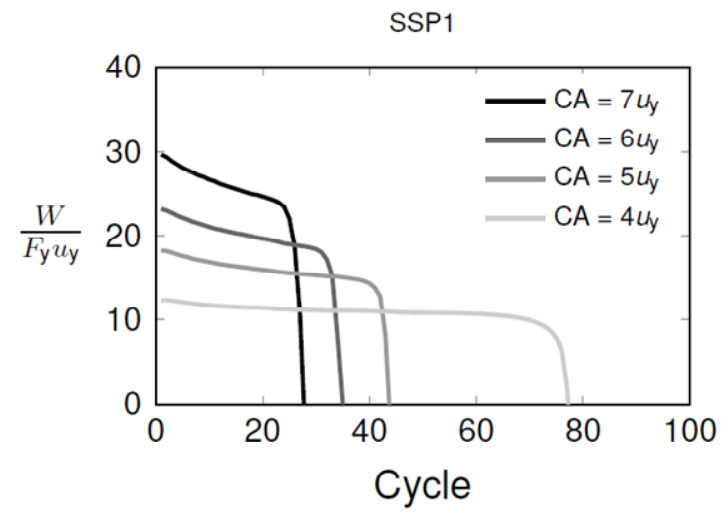

(a)

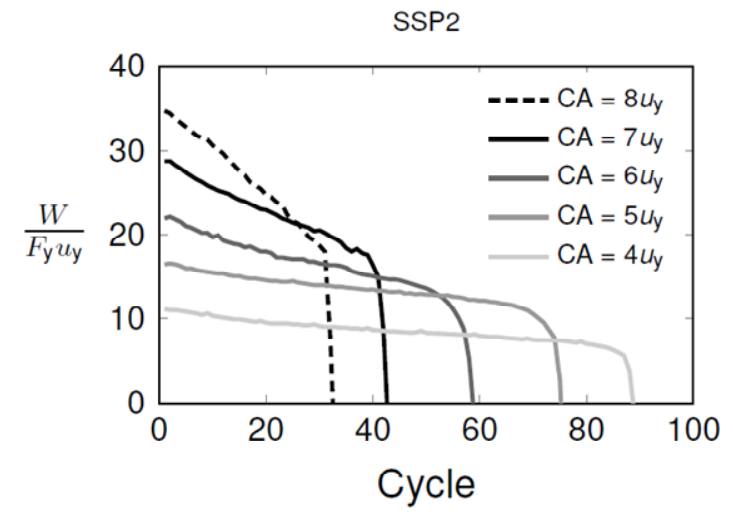

(b)

Fig. 10. Energy dissipation in CA tests: (a) SSP1; and (b) SSP2 


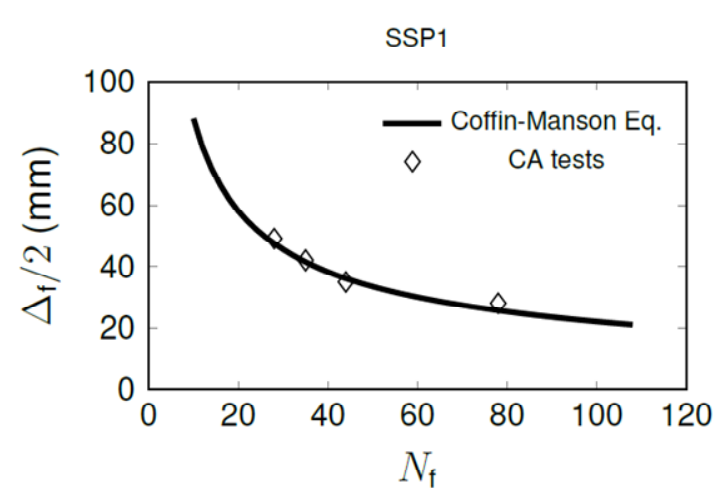

(a)

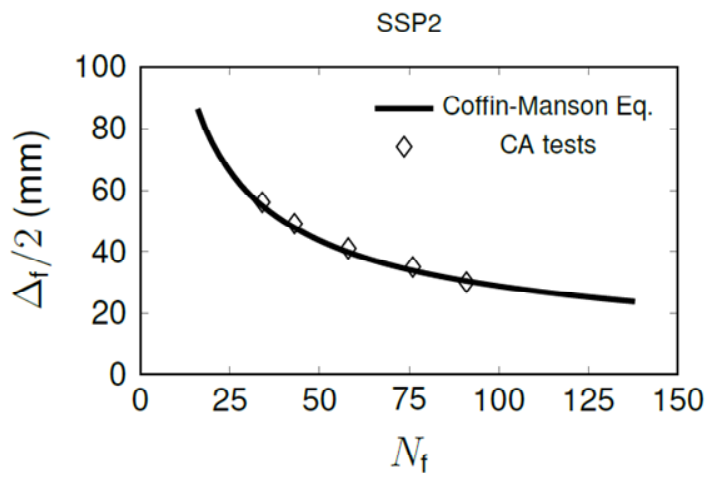

(b)

Fig. 11. Imposed amplitude versus number of cycles to failure relationship: (a) SSP1; and (b) SSP2 


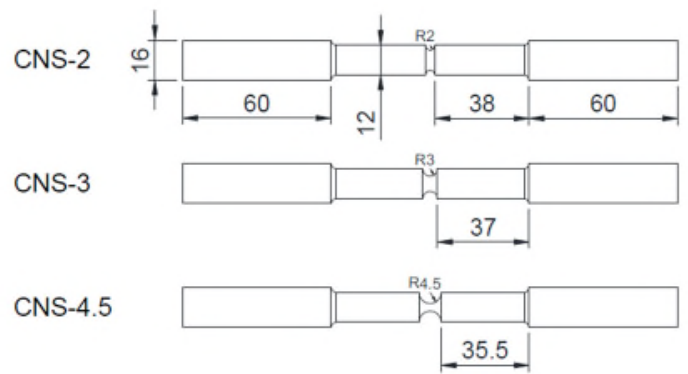

(a)

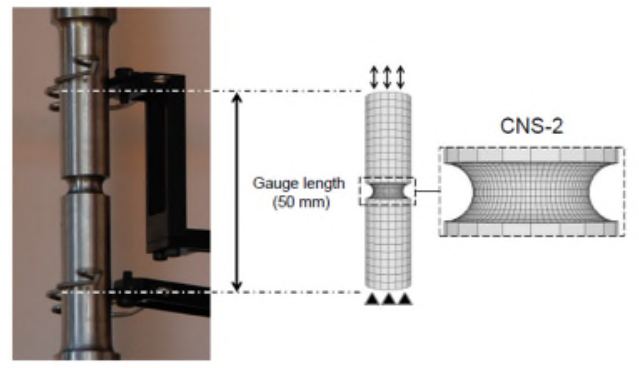

(b)

Fig. 12. (a) CNS geometry; (b) FEM model of CNS-2 (gauge length) 


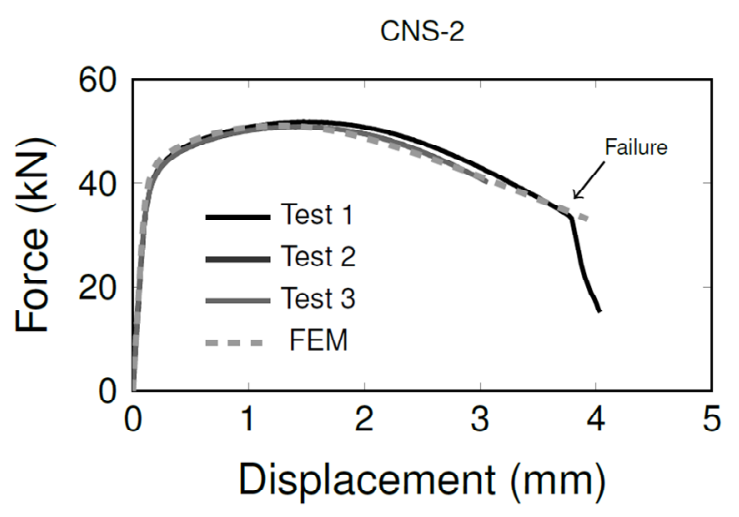

(a)

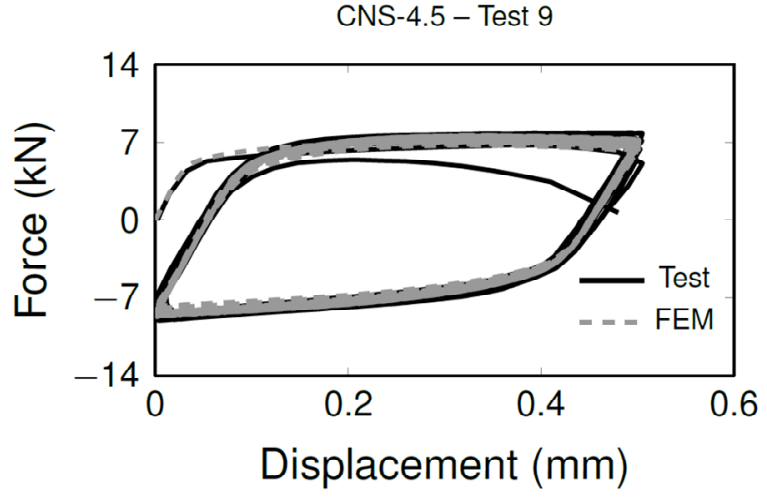

(b)

Fig. 13. Experimental-FEM comparison of CNS response (simulation without fracture definition): (a) monotonic; and (b) cyclic 


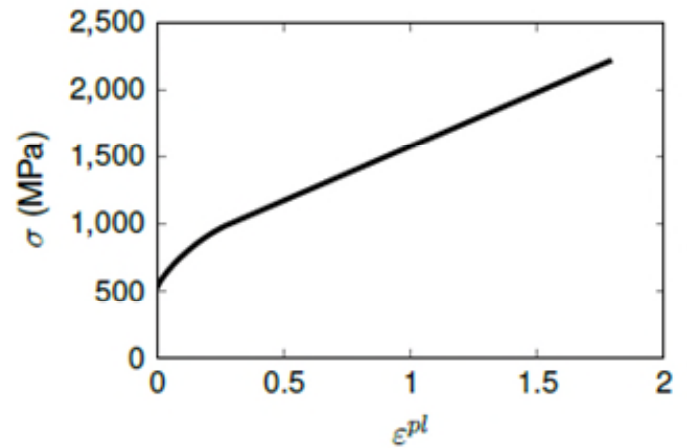

Fig. 14. True stress-true plastic strain curve of SSD. 


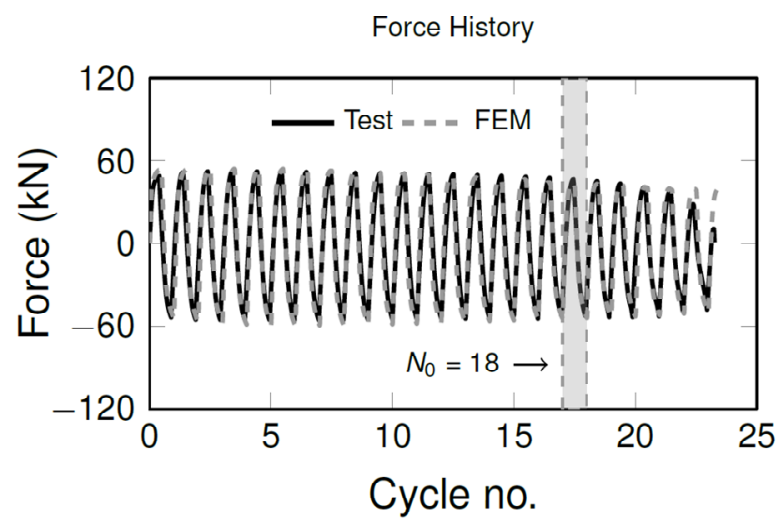

(a)

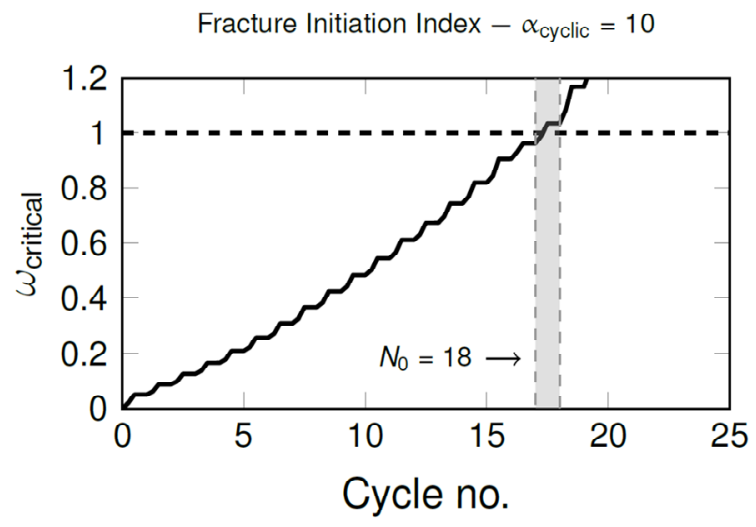

(b)

Fig. 15. CNS-2 (test 3): (a) experimental versus FEM force history; and (b) $\omega_{\text {critical }}$ evolution up to fracture. The grey vertical area denotes fracture initiation 


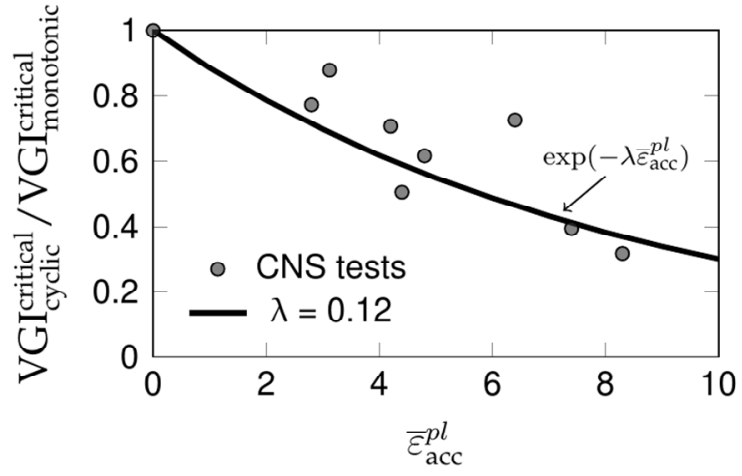

Fig. 16. Calibration of $\lambda$ based on $\mathrm{VGI}_{\text {cyclic }}^{\text {critical }} / \mathrm{VGI}_{\text {monotonic }}^{\text {critical }}$ ratios and associated $\bar{\varepsilon}_{\text {acc }}^{p l}$ values from CNS tests 


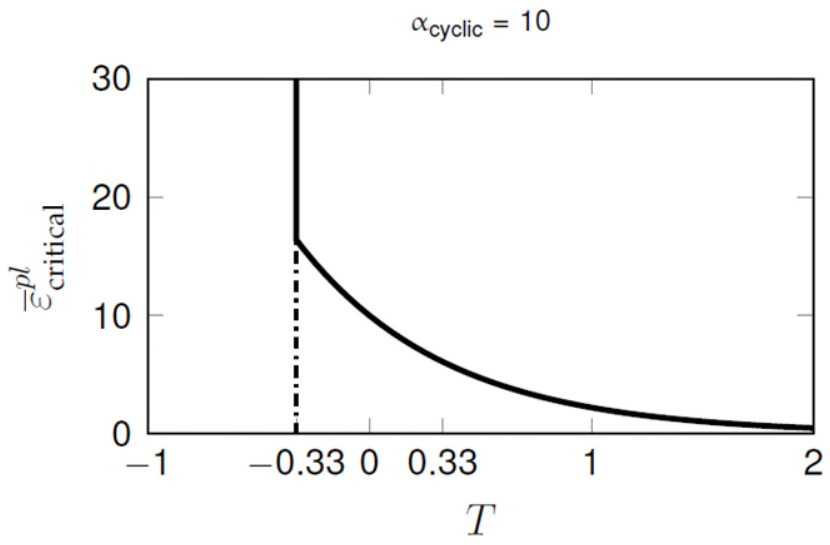

Fig. 17. Abaqus fracture initiation model calibrated for ultra-low cycle fatigue 
CNS-2 - Test 3

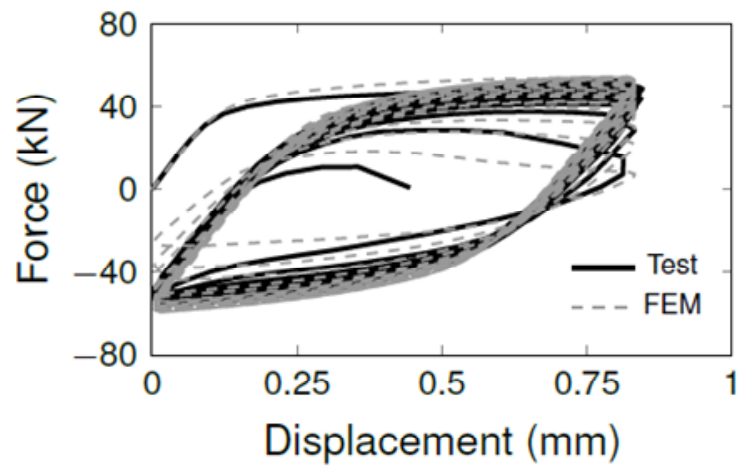

CNS-3 - Test 6

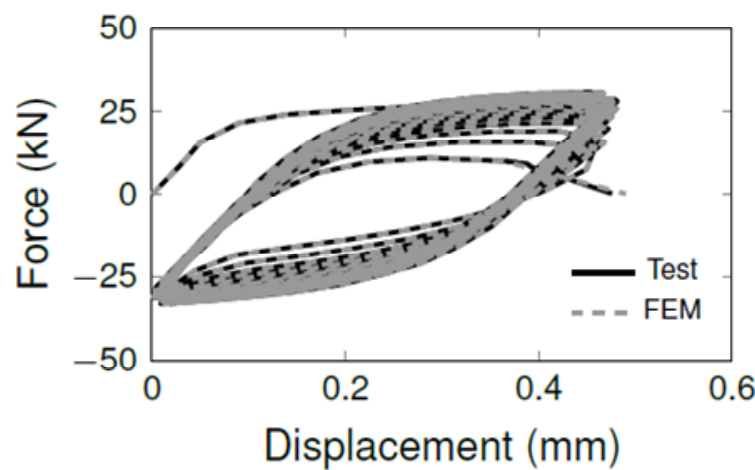

CNS-4.5 - Test 9

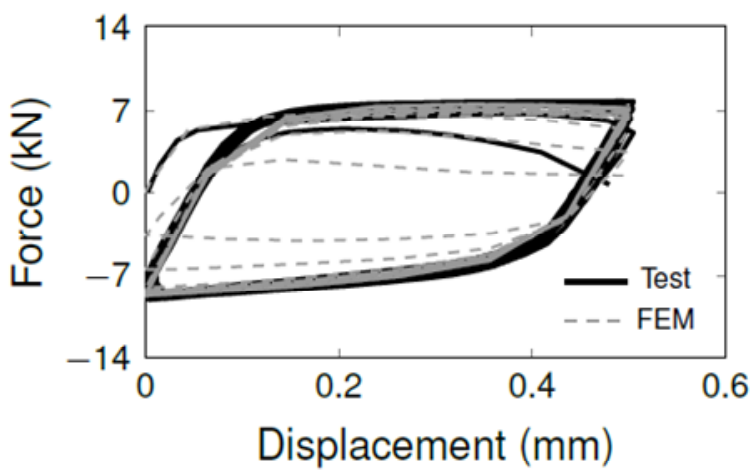

(a)

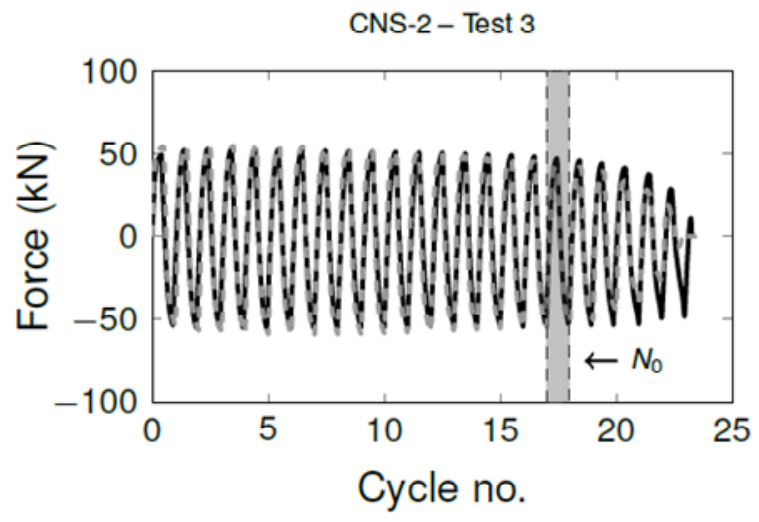

CNS-3 - Test 6

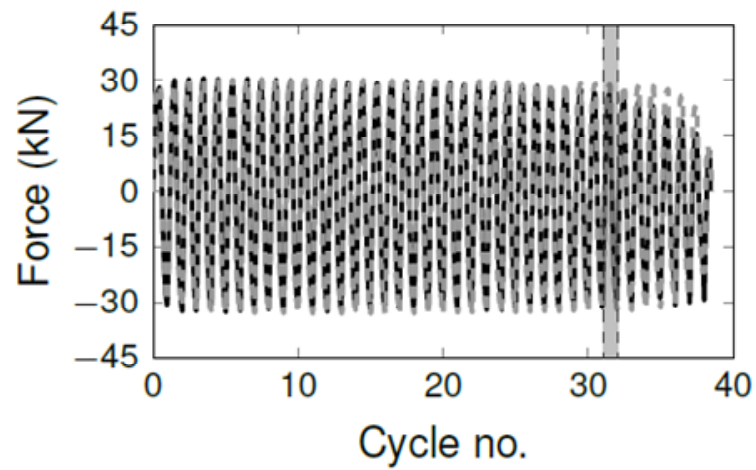

CNS-4.5 - Test 9

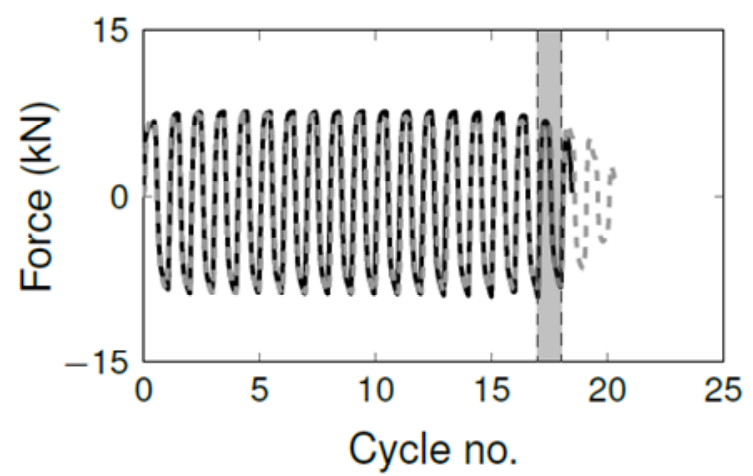

(b)

Fig. 18. Comparison of the experimental and numerical results: (a) force-displacement behavior; and (b) force history with indication of experimental ductile fracture initiation (cycle no.) 


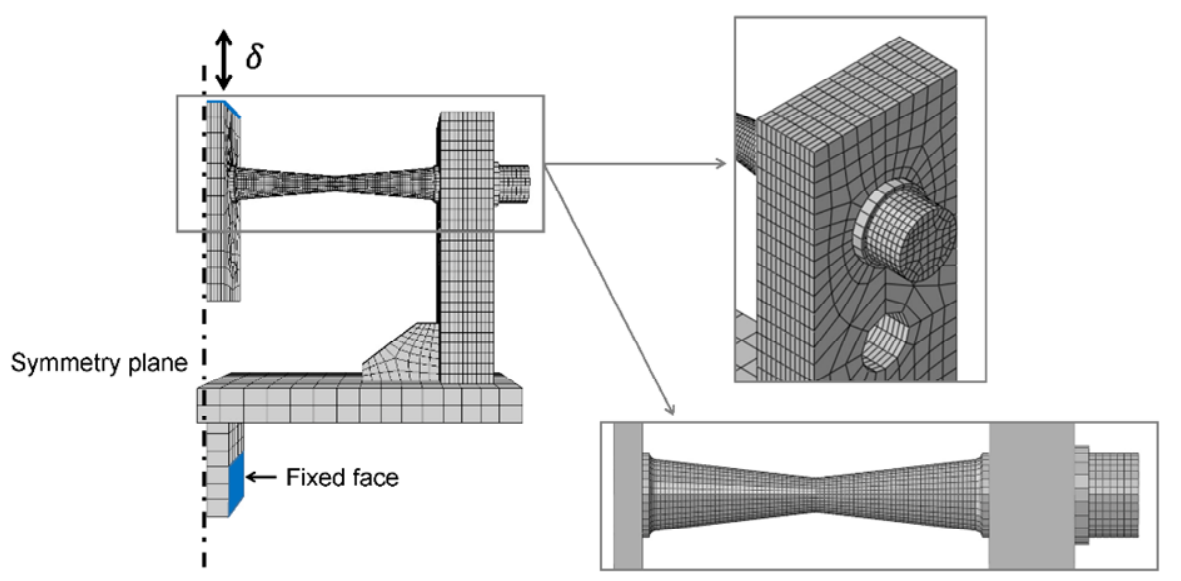

Fig. 19. SSP1 FEM model: mesh discretization and boundary conditions 


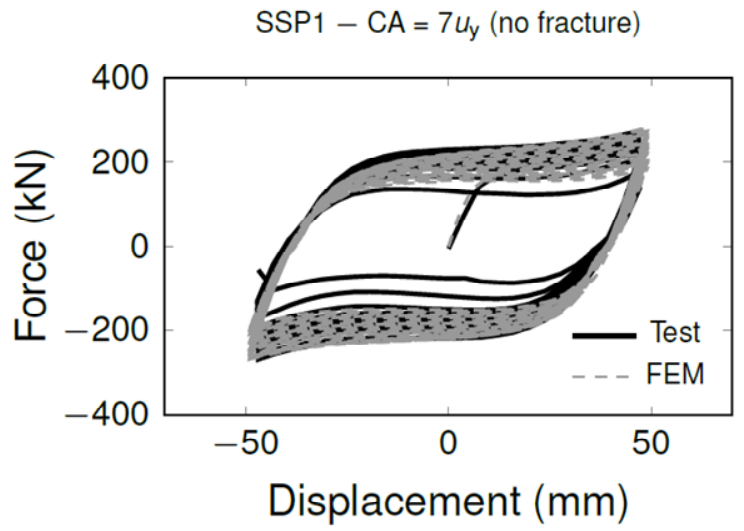

(a)

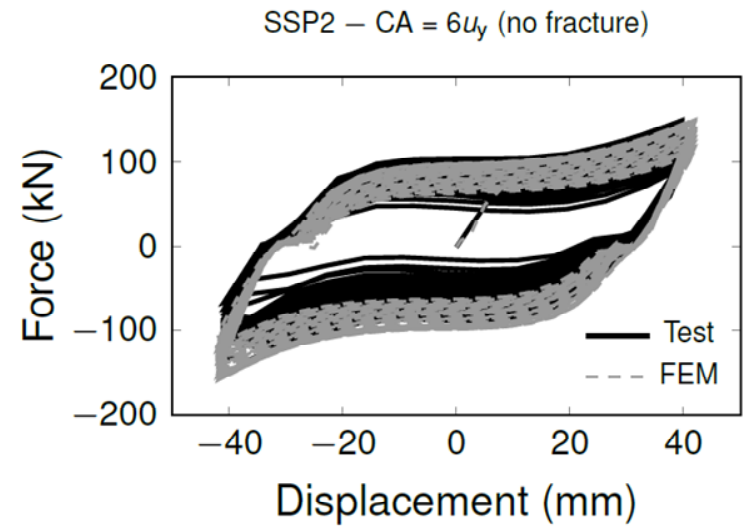

(b)

Fig. 20. Experimental and numerical (without fracture criteria) hysteresis: (a) SSP1 (CA = 7uy); and (b) SSP2 (CA = 6uy) 


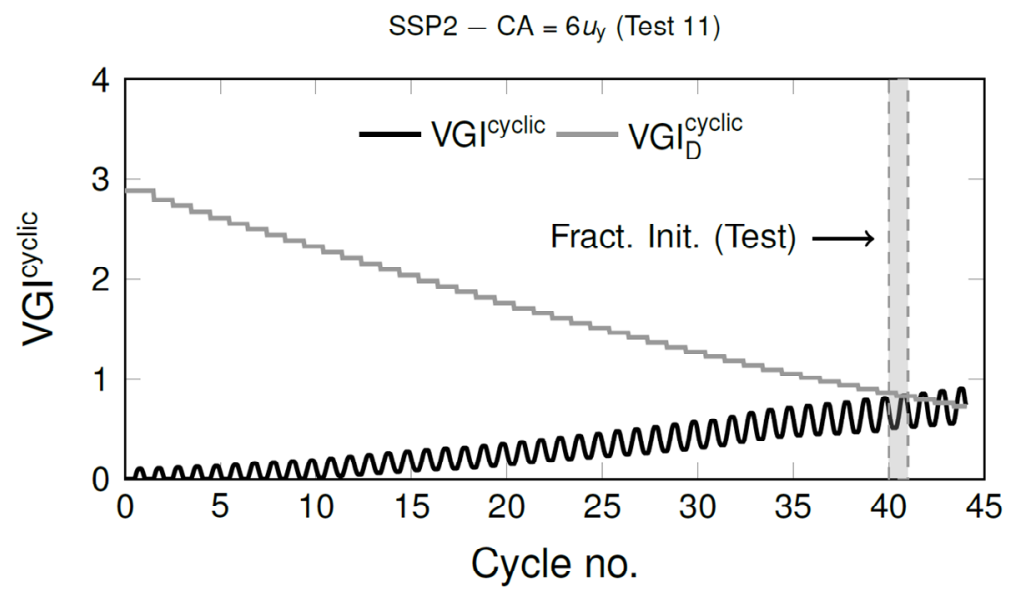

Fig. 21. $\mathrm{CVGM}$ fracture prediction in $\mathrm{SSP} 2$ under $\mathrm{CA}=6 u_{\mathrm{y}}$ 

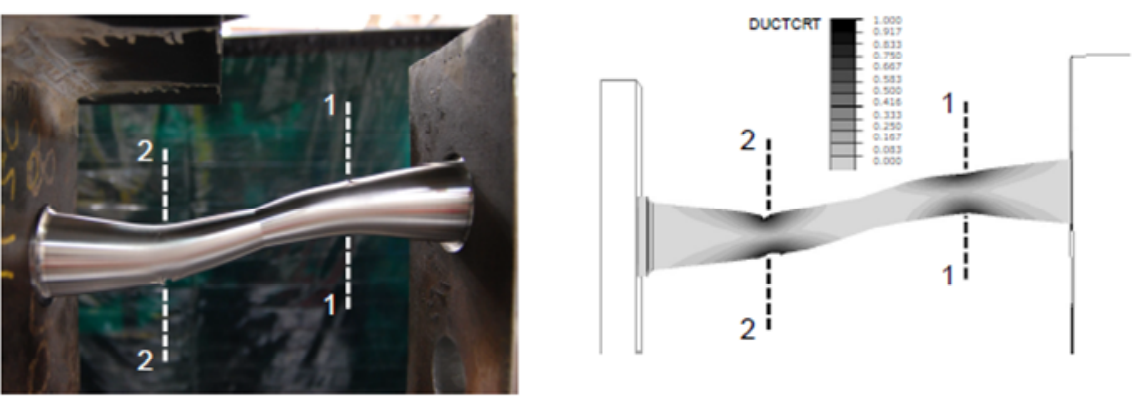

Test - SSP2

FEM model - SSP2
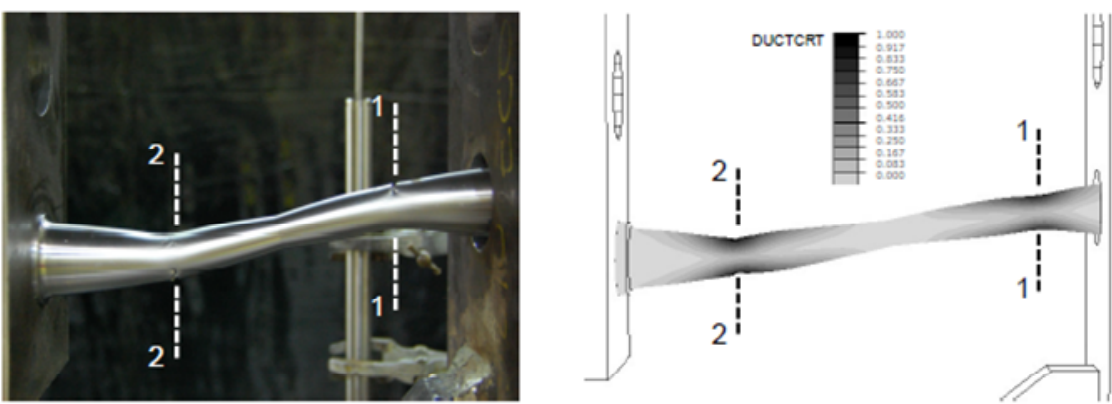

Fig. 22. Experimental and numerical fracture locations in SSPs 


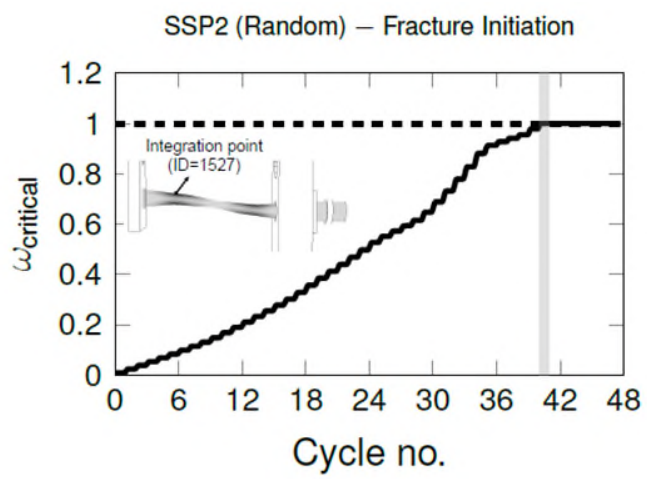

(a)

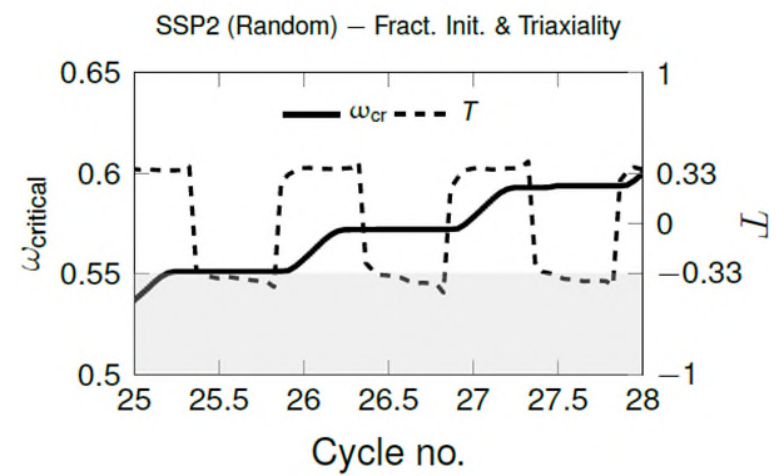

(b)

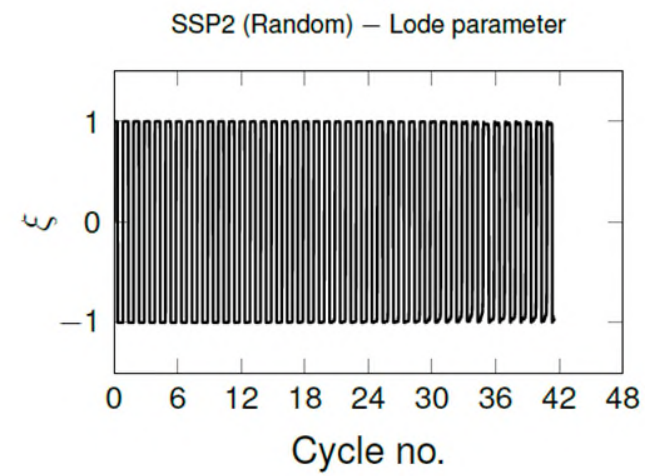

(c)

Fig. 23. SSP2 Random test: (a) fracture initiation index evolution; (b) fracture index and triaxiality evolution; and (c) Lode parameter evolution. 

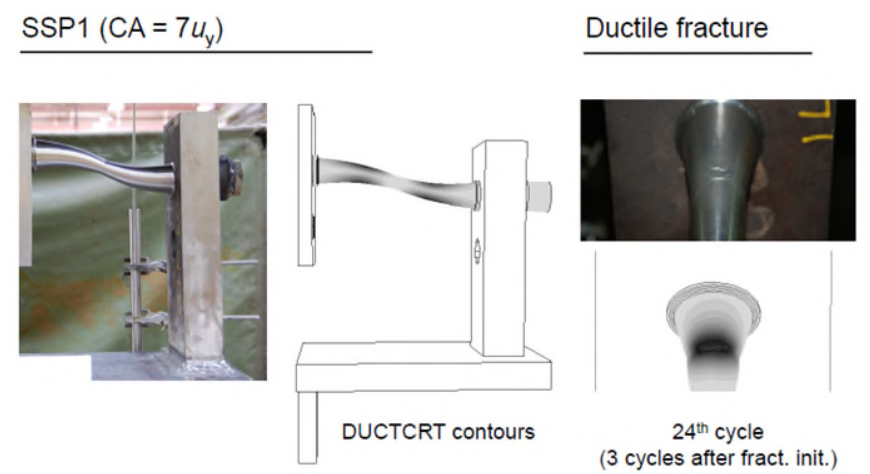

(a)
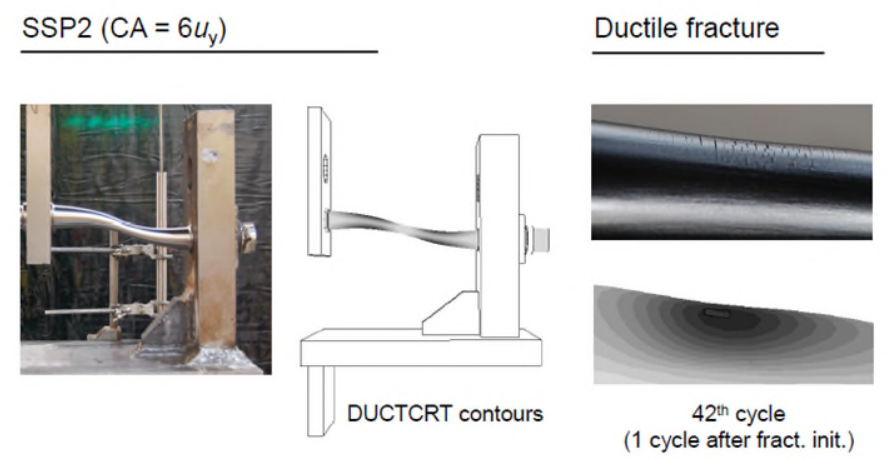

Failure

(b)

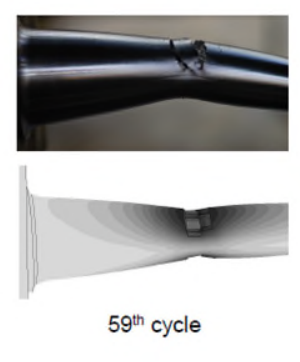

Fig. 24. Comparison of experimental and numerical ductile fracture evolution in section 2 for: (a) SSP1; and (b) SSP2. 


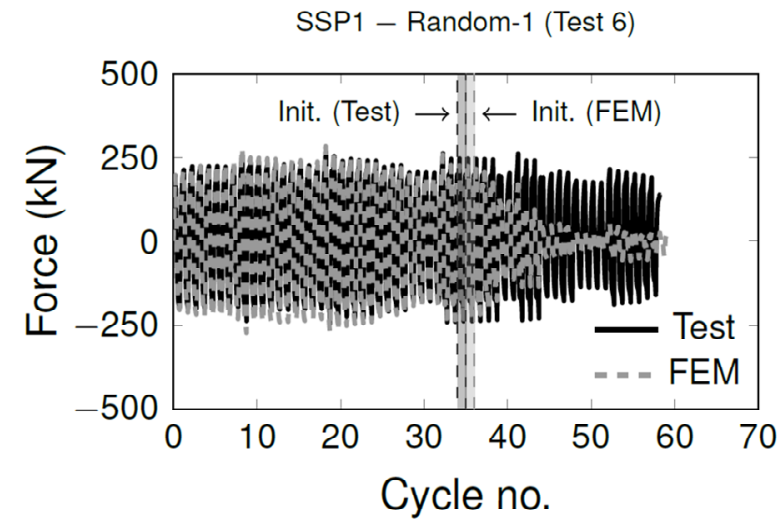

(a)

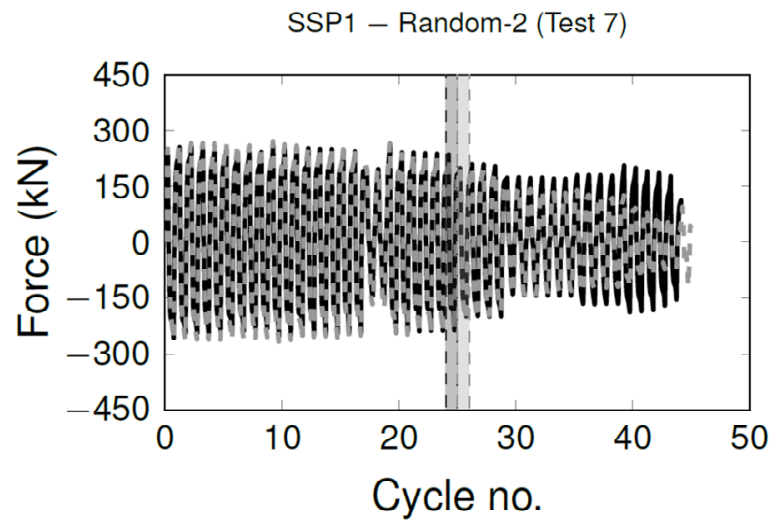

(b)

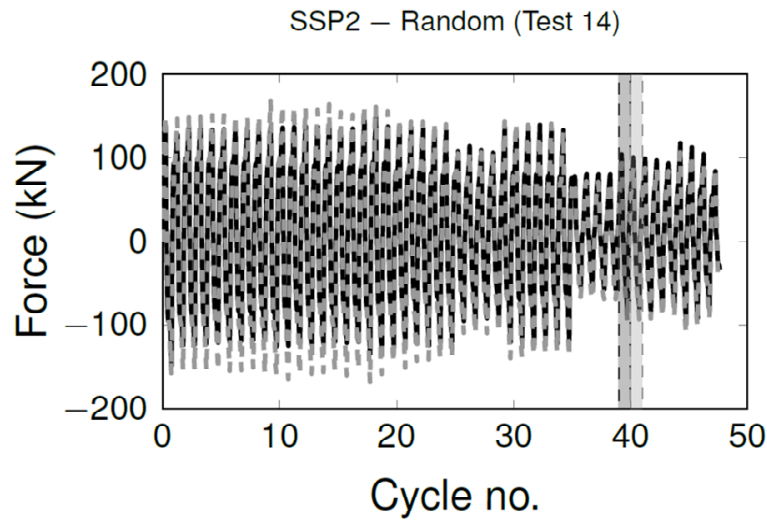

(c)

Fig. 25. Experimental-numerical force histories of SSPs: random tests. 